\title{
On a Semiclassical Limit of Loop Space Quantum Mechanics
}

\author{
Partha Mukhopadhyay \\ The Institute of Mathematical Sciences, C.I.T. Campus, Taramani, Chennai 600113, India \\ Correspondence should be addressed to Partha Mukhopadhyay; parthamu@imsc.res.in
}

Received 28 February 2013; Accepted 10 April 2013

Academic Editors: A. Belhaj, A. Konechny, A. Koshelev, and L. Pando Zayas

\begin{abstract}
Copyright (C) 2013 Partha Mukhopadhyay. This is an open access article distributed under the Creative Commons Attribution License, which permits unrestricted use, distribution, and reproduction in any medium, provided the original work is properly cited.
\end{abstract}

\begin{abstract}
Following earlier work, we view two-dimensional nonlinear sigma model as single particle quantum mechanics in the free loop space of the target space. In a natural semiclassical limit of this model, the wavefunction localizes on the submanifold of vanishing loops. One would expect that the semiclassical expansion should be related to the tubular expansion of the theory around the submanifold and effective dynamics on the submanifold is obtainable using Born-Oppenheimer approximation. We develop a framework to carry out such an analysis at the leading order. In particular, we show that the linearized tachyon effective equation is correctly reproduced up to divergent terms all proportional to the Ricci scalar. The steps are as follows: first we define a finite dimensional analogue of the loop space quantum mechanics (LSQM) where we discuss its tubular expansion and how that is related to a semiclassical expansion of the Hamiltonian. Then we study an explicit construction of the relevant tubular neighborhood in loop space using exponential maps. Such a tubular geometry is obtained from a Riemannian structure on the tangent bundle of target space which views the zero-section as a submanifold admitting a tubular neighborhood. Using this result and exploiting an analogy with the toy model, we arrive at the final result for LSQM.
\end{abstract}

\section{Introduction and Summary}

Strings in curved background is a well-studied problem [1,2]. Usually the semiclassical expansion is formulated using the background field method of quantum field theory (QFT) in Lagrangian framework [3-6]. An attractive feature of this formulation is the use of Riemann normal coordinate (RNC) [7] expansion. This enables one to keep the Riemannian structure of the target manifold $\mathscr{M}$ manifest.

Although it is not usually used for QFT computations, Hamiltonian framework, on the other hand, is conceptually appealing. It is natural to view the two-dimensional nonlinear sigma model (NLSM) under consideration as a single particle relativistic quantum mechanics in the infinite dimensional free loop space $\mathscr{L} \mathscr{M}$ corresponding to $\mathscr{M}[8-$ 13]. In $[14,15]$ we discussed a framework of describing this quantum mechanics, hereafter called loop space quantum mechanics (LSQM), for the bosonic sigma model in terms of general coordinates in $\mathscr{L} \mathscr{M}$ keeping the infinite dimensional Riemannian structure manifest. This may be viewed as a formal $\hbar$-deformation of the classical theory as divergences are present in the form of infinite dimensional traces. The problem of regularizing these divergences was emphasized earlier in $[8,9,16]$. As a first step towards this direction, in this paper we discuss a semiclassical limit of LSQM and motivate the use of Fermi normal coordinate (FNC) $[17,18]$ expansion describing the tubular neighborhood of $\mathscr{M}$ when it is viewed as the submanifold of vanishing loops embedded in $\mathscr{L} \mathscr{M}$ (the view of studying $\mathscr{M}$ through its embedding in $\mathscr{L} \mathscr{M}$ was considered earlier by Witten in [9]).

We now roughly describe the general idea. One expects that in $\hbar=\alpha^{\prime} \rightarrow 0$ limit the worldsheet theory should reduce to a theory of particles in $\mathscr{M}$. One also notices that LSQM has a potential which minimizes to zero on the submanifold of zero loops. Therefore, a natural semiclassical limit is given by the situation where the wavefunction localizes on this submanifold. The general idea is to use Born-Oppenheimer type approximation to adiabatically decouple the longitudinal (slow) and transverse (fast) degrees of freedom (This is similar in spirit to the discussion of degenerate Morse theory in [8]. As explained below, we will study this problem in more detail following certain other literature.) and finally to compute the effective theory on $\mathscr{M} \hookrightarrow \mathscr{L} \mathscr{M}$ order by order in $\hbar$. 
A complete understanding of the above procedure requires several technical questions to be answered. Some of them are as follows.

(1) How to perform tubular expansion of tensors around a submanifold embedded in a higher dimensional ambient space?

(2) Given a suitable quantum mechanical problem in the ambient space, how to set up the relevant semiclassical expansion of the Hamiltonian which relates to the above tubular expansion? How to get an effective theory on the submanifold?

(3) Given the understanding of the above questions in a finite dimensional case, how to apply them to our present context of loop space?

We will discuss all the above three topics successively and our final goal will be to derive the linearized effective equation for the tachyon fluctuation at leading order in $\alpha^{\prime}$-expansion. We will show that our analysis correctly reproduces the known result up to divergent terms all proportional to the Ricci scalar of $\mathscr{M}$. Below we briefly discuss these topics to indicate how this result will be arrived at. This will also clarify relation to other works in the literature.

The first question is discussed in Section 2 (and in Appendix A). Here we explain our basic set up for a finite dimensional submanifold embedding, introduce FNC, and review the results of [19]. In [19], by generalizing the techniques of [20], we find all order FNC-expansion of vielbein components in the neighborhood of a submanifold (say $M$ ) embedded in a pseudo-Riemannian ambient space (say $L)(M$ and $L$ are our finite dimensional analogues of $\mathscr{M}$ and $\mathscr{L} \mathscr{M}$, resp.). The expansion coefficients are given by certain tensors of $L$, all evaluated at $M \hookrightarrow L$. For vielbein these tensors are given by combinations of various powers of the curvature, their covariant derivatives and spin connection. For the rest of our analysis the FNC-expansion of the metric tensor up to quadratic order, as given in (3), will be crucially used.

To address the second question we consider a finite dimensional analogue of LSQM in Section 3. The analysis in this section is along the line of what is usually known as constrained quantum system in the literature. A partial list of references is [21-28]. Here one considers a nonrelativistic classical system in an ambient space with a potential that tries to confine the motion into a submanifold. The idea is to realize this constraint at the quantum mechanical level through localization of wavefunction. This is done by rescaling the model with certain tunable parameter (e.g., representing the strength of the restoring force) in such a way that makes the transverse directions fast in the BornOppenheimer sense when the parameter is small. In our case the tunable parameter is the scale $\hbar$ and therefore the procedure gives a semiclassical expansion of the theory. In Section 3 we give precise definition of the potential of our model and the procedure leading to semiclassical expansion of the Hamiltonian. This shows how the contribution at a given order in $\hbar$ is related to tubular expansion of various geometric quantities at different orders. Finally, we define and compute an analogue of linearized tachyon effective equation at leading order in $\hbar$-expansion within this toy model.
The usefulness of this study lies in the fact that it is free of divergences. Moreover, as hinted in the next paragraph, there exists an analogy which can be exploited to translate the end results of the toy model to the case of LSQM. Once this is done it exhibits the pattern of divergences that are expected in the actual LSQM computations. This is how we arrive at the final result for the tachyon effective equation as mentioned earlier.

We now turn to the question of how to translate the results of finite dimensional model to the case of LSQM which is the content of the third question. Given that the theory is being expanded around a submanifold, such results are in general expressed in terms of various tubular expansion coefficients which are tensors of the ambient space evaluated on the submanifold. Since the Riemannian structure of $\mathscr{L} \mathscr{M}$ is induced from that of $\mathscr{M}$, one would expect that all the relevant tubular expansion coefficients should be related to certain intrinsic geometric data of $\mathscr{M}$. Finding such relations for the metric-expansion coefficients up to quadratic order will be the precise quantitative question addressed in Section 4. There are several technical steps to be followed in order to arrive at the final result which we explain in a self-contained manner in Section 4 (In more technical terms, the final goal of Section 4 is to develop a precise understanding of the metric-expansion given in (3) in the context of loop space. This is done by suitably constructing (1) the tubular neighborhood of $\mathscr{M} \hookrightarrow \mathscr{L} \mathscr{M}$ (content of Section 4.1) and (2) the FNC in $\mathscr{L} \mathscr{M}$ (content of Section 4.2). A construction of the relevant tubular neighborhood appeared before in [29]. Although the general ideas are similar, our detailed construction is different and chosen to suit our purpose of constructing FNC. In particular, [29] uses a method of embedding $\mathscr{M}$ in a higher dimensional Euclidean space, whereas we use exponential maps. This enables us to relate the tubular geometry in $\mathscr{L} \mathscr{M}$ to a Riemannian structure in $T \mathscr{M}$.). Once these relations are known, one can use the precise analogy between the toy model and LSQM to translate results of Section 3. This will be discussed in Section 5. We conclude in Section 6 with some future directions. A brief note on loop space and LSQM that will be relevant for our discussion has been given in Appendix C. Appendices B and $\mathrm{D}$ contain some technical details.

\section{Tubular Expansion of Metric up to Quadratic Order}

Here we describe the basic set up for submanifold embedding that will be used throughout the paper. We consider a $D$ dimensional subspace $M$ embedded in a higher dimensional (pseudo) Riemannian space $L$ of dimension $d$. We adopt the following notations. Greek indices $(\alpha, \beta, \ldots)$ run over $D$ dimensions, capital Latin indices $(A, B, \ldots)$ run over $(d-$ $D)$ dimensions, and small Latin indices $(a, b, \ldots)$ over all $d$ dimensions. The coordinates of $L$ will be denoted by $z^{a}=$ $\left(x^{\alpha}, y^{A}\right)$ where $x^{\alpha}$ is a general coordinate system in $M$. Indices kept inside parenthesis will refer to noncoordinate basis, $\eta_{(a b)}$ being the diagonal matrix with the indictors as diagonal elements. 
In [17] Florides and Synge (FS) proved existence of certain submanifold based coordinate system, called FNC in modern literature [18], which satisfies special coordinate conditions. In the special case where $M$ is a point, FNC reduces to RNC. The FS coordinate conditions can be described as follows. Equation for the submanifold is given by

$$
y^{A}=0 \text {. }
$$

The metric components of $L$, denoted by $g_{a b}(z)$, satisfy the following equations:

$$
\begin{gathered}
g_{a B}(z) y^{B}=\bar{g}_{a B}(x) y^{B}, \\
\bar{g}_{\alpha B}(x)=0, \\
\bar{g}_{A B}(x)=\eta_{A B},
\end{gathered}
$$

where $\bar{g}_{a b}(x)=g_{a b}(x, y=0)$. As a general rule, we use the lower case symbols to denote the geometric quantities of $L$ and the same symbols with bars to denote the same quantities restricted to the submanifold. With this convention in mind we will refrain from explicitly writing down the arguments of such quantities most of the time.

The results for the expansion of the metric components away from the submanifold that will be relevant for us later are given by

$$
\begin{aligned}
& g_{\alpha \beta}= G_{\alpha \beta}+\bar{s}_{\alpha \beta C} y^{C} \\
&+\left(\bar{\omega}_{\alpha}{ }^{\gamma}{ }_{C} \bar{\omega}_{\beta \gamma D}+\bar{\omega}_{\alpha{ }^{B}}{ }^{B} \bar{\omega}_{\beta B D}+\bar{r}_{\alpha C D \beta}\right) y^{C} y^{D} \\
&+O\left(y^{3}\right), \\
& g_{\alpha B}=\bar{\omega}_{\alpha B C} y^{C}+\frac{2}{3} \bar{r}_{\alpha C D B} y^{C} y^{D}+O\left(y^{3}\right), \\
& g_{A B}=\eta_{A B}+\frac{1}{3} \bar{r}_{A C D B} y^{C} y^{D}+O\left(y^{3}\right),
\end{aligned}
$$

where $\omega_{a}{ }^{(b)}{ }_{(c)}$ are components of the connection one-form of $L$ (noncoordinate indices are converted to coordinate indices with the use of vielbein as usual), $r_{a c d b}$ is the covariant Riemann curvature tensor (we follow the same convention for the curvature as in [30]), and

$$
\bar{s}_{\alpha \beta C}=\bar{\omega}_{\alpha \beta C}+\bar{\omega}_{\beta \alpha C},
$$

is the second fundamental form of the submanifold embedding [31].

We obtain (3) from a closed form expression for the expansion of vielbein which is derived in [19]. Although the details of this result will not be directly used in this work, there will be some relevance in the discussion of Section 4. We therefore summarize the main results of [19] in Appendix A.

\section{Finite Dimensional Analogue of Loop Space Quantum Mechanics}

In this section we will consider a finite dimensional analogue of LSQM in the framework discussed in $[14,15]$. In Section 3.1 we will define the model and its semiclassical expansion. The analogue of linearized effective equation for tachyon fluctuation at leading order will be derived in Section 3.2. Our discussion below will be done without any reference to LSQM. We will come back to the analogy later in Section 5.

3.1. Definition of Model and Semiclassical Expansion of Hamiltonian. All our notations used in Section 2 will be valid in this section. We consider a nonrelativistic quantum mechanical system whose configuration space is given by $L$. Hence it is assumed (only in this section) to have Euclidean signature. The Hamiltonian of the system is given by the standard expression

$$
\begin{aligned}
\left\langle\chi^{\prime}\left|H^{\mathrm{pre}}\right| \psi^{\prime}\right\rangle & =\int d w \chi^{\prime *}(z) \mathscr{H}^{\mathrm{pre}} \psi^{\prime}(z), \\
\mathscr{H}^{\mathrm{pre}} & =-\frac{\hbar^{2}}{2} \mathscr{D}^{2}+V,
\end{aligned}
$$

where $d w=d z \sqrt{g}$ is the invariant measure, $\mathscr{D}^{2}$ is the Laplacian of $L$, and $V$ is a potential. We will consider $V$ to be confining to the submanifold $M \hookrightarrow L$ (a more precise definition will follow). We must define what we mean by performing a semiclassical expansion such that in the semiclassical limit the wavefunction collapses on the submanifold. This is a procedure given by the following steps (Various other, but similar, procedures have been discussed in the literature indicated earlier. Our procedure is adopted to suit LSQM.)

(1) Submanifold Based Description. Given $H^{\text {pre }}$ as in (5), we first move to a submanifold based description where the natural measure is given by $d y d x \sqrt{G}$ instead of $d w \sqrt{g}$ (Recalling our notations introduced in Section $2, d x \sqrt{G}$ is the invariant measure on the submanifold with respect to the induced metric.). As discussed in Appendix B, this is done by performing certain rescaling of the wavefunction so that the same matrix element in (5) is given in terms of the transformed Hamiltonian, which we call $H^{\text {sub }}$, and transformed wavefunctions (unprimed) as

$$
\left\langle\chi\left|H^{\mathrm{sub}}\right| \psi\right\rangle=\int d y d x \sqrt{G} \chi^{*}(z) \mathscr{H}^{\mathrm{sub}} \psi(z),
$$

where the expression for $\mathscr{H}^{\text {sub }}$ can be found in (B.2).

(2) Tubular Expansion. Next we tubular expand $\mathscr{H}^{\text {sub }}$ to write

$$
\left\langle\chi\left|H^{\text {sub }}\right| \psi\right\rangle=\sum_{n=0}^{\infty} \int d y d x \sqrt{G} \chi^{*}(z) \mathscr{H}_{n}^{\text {sub }} \psi(z),
$$

where we adopt the following notation: $X_{n}$ is the contribution at $O\left(y^{n}\right)$ in the tubular expansion of $X$. Explicit result for $\mathscr{H}_{n}^{\text {sub }}$ is given in (B.4), (B.5), and (B.6).

(3) Definition of $V$. The potential $V$ is confining to the submanifold $M$ whose embedding satisfies the following property:

$$
\bar{\omega}_{\alpha \beta C}=0
$$


As a result the second fundamental form in (4) vanishes and therefore $M$ is totally geodesic [31]. Furthermore, the transverse profile of $V$ is given by the following expression in FNC (We will display the summation over indices explicitly, as we have done in (9), whenever Einstein summation convention will not be valid.):

$$
V(x, y)=\frac{1}{2} \sum_{A, B} \epsilon_{A} \epsilon_{B} g_{A B} y^{A} y^{B},
$$

where $\epsilon_{A}$ is positive definite (Notice that due to the presence of the $\epsilon$-factors in the potential, general covariance of the ambient manifold is broken down to that of the submanifold even at the classical level. Without such factors $V$ will be a scalar under the full diffeomorphism of $L$, but will not have a nontrivial tubular expansion due to the coordinate condition in (2). As will be explained in Section 5, general covariance gets broken in LSQM only due to the semiclassical limit.).

(4) Definition of Semiclassical Expansion. Define a rescaled Hamiltonian,

$$
H=\frac{1}{\hbar} H^{\text {sub }} .
$$

Semiclassical expansion of $H$ is given by rescaling the transverse coordinates as

$$
y^{A} \longrightarrow \sqrt{\frac{\hbar}{\epsilon_{A}}} y^{A}
$$

in the tubular expansion of $H$. This gives

$$
\langle\chi|H| \psi\rangle=\sum_{n=0}^{\infty} \hbar^{n / 2} \int d y d x \sqrt{G} \chi^{*}(z)^{\epsilon} \mathscr{H}^{(n)} \psi(z),
$$

where we have used the following notation:

$$
{ }^{\epsilon} X=\left.X\right|_{y^{A} \rightarrow y^{A} / \sqrt{\epsilon_{A}}} .
$$

The expression for ${ }^{\epsilon} \mathscr{H}^{(n)}$ can be found in (B.7). For the rest of our analysis we will restrict the expansion in (12) up to $O(\hbar)$. Explicit computation using the metric-expansion in (3) yields the following results:

$$
\begin{gathered}
\epsilon_{\mathscr{H}^{(0)}}=\frac{1}{2} \sum_{A, B} \sqrt{\epsilon_{A} \epsilon_{B}}\left(-\eta^{A B} \partial_{A} \partial_{B}+\eta_{A B} y^{A} y^{B}\right) \\
=\sum_{A, B} \sqrt{\epsilon_{A} \epsilon_{B}} \eta_{A B} a^{\dagger A} a^{B}+\frac{1}{2} \sum_{A} \epsilon_{A}, \\
\epsilon \mathscr{H}^{(1)}=0, \\
\epsilon_{\mathscr{H}^{(2)}=}-\frac{1}{2}\left(\nabla^{\alpha}+i \bar{\omega}^{\alpha A B}{ }^{\alpha} \Lambda_{A B}\right)\left(\nabla_{\alpha}+i \bar{\omega}_{\alpha}{ }^{C D}{ } \Lambda_{C D}\right) \\
-\frac{1}{4} \bar{r}_{\|}-\frac{1}{12} \bar{r}_{\perp}+\frac{1}{6} \bar{r}^{A B C D \epsilon} \Lambda_{A B}{ }^{\epsilon} \Lambda_{C D} \\
+\frac{1}{6} \sum_{A, B} \sqrt{\frac{\epsilon_{A} \epsilon_{B}}{\epsilon_{C} \epsilon_{D}}} \bar{r}_{A C D B} y^{A} y^{C} y^{D} y^{B},
\end{gathered}
$$

where in the first equation we have defined annihilation and creation operators,

$$
a^{A}=\frac{1}{\sqrt{2}}\left(\eta^{A B} \partial_{B}+y^{A}\right), \quad a^{\dagger A}=\frac{1}{\sqrt{2}}\left(-\eta^{A B} \partial_{B}+y^{A}\right),
$$

respectively, such that $\left[a^{A}, a^{\dagger B}\right]=\eta^{A B}$. In the last equation $\nabla_{\alpha}$ denotes the covariant derivative with respect to the induced metric $G_{\alpha \beta}(x)$ on $M$. The other new notations introduced in these equations are as follows:

$$
\begin{gathered}
\bar{r}_{\|}=\bar{r}_{\alpha B}^{\alpha B}, \quad \bar{r}_{\perp}=\bar{r}_{A B}^{A B}, \\
{ }^{\epsilon} \Lambda_{A B}=-\frac{i}{2}\left(\sqrt{\frac{\epsilon_{B}}{\epsilon_{A}}} \eta_{A C} y^{C} \partial_{B}-\sqrt{\frac{\epsilon_{A}}{\epsilon_{B}}} \eta_{B C} y^{C} \partial_{A}\right) .
\end{gathered}
$$

As noted in the literature, $\Lambda_{A B}$ is the angular momentum operator in the transverse space and $\bar{\omega}_{\alpha A B}$ is analogous to a nonabelian $(S O(d-D))$ Berry connection [32]. As a mathematical exercise, our results in (14) are equivalent to a case discussed in [25] except for the last term in the last equation which comes from the tubular expansion of our potential.

The reasons why the above procedure correctly captures our general idea of Born-Oppenheimer type approximation and localization of wavefunction are as follows. The $\hbar$ dependent rescaling in (11) makes the transverse coordinates $y$ fast in the Born-Oppenheimer sense. As a result, the leading order harmonic oscillator Hamiltonian, that is, ${ }^{\epsilon} \mathscr{H}^{(0)}$, is independent of the slow coordinates $(x)$. Therefore, the transverse and longitudinal dynamics decouple. Moreover, ${ }^{\epsilon} \mathscr{H}^{(0)}$ is $\hbar$-independent. The wavefunctions fall to zero at large values of the rescaled coordinates. At leading order this corresponds to arbitrary finite values of the original transverse coordinates indicating that the wavefunctions are localized.

3.2. Analogue of Linearized Tachyon Effective Equation at Leading Order. Here we consider the transverse degrees of freedom to be frozen in the harmonic oscillator ground state and derive the effective Hamiltonian, as will be defined in (22) below, for the longitudinal degree of freedom at the leading order. This will give us the linearized tachyon effective equation (see (24)) at this order. We will explain this analogy later in Section 5.

The wavefunction under consideration is

$$
\psi_{0}(x, y)=T(x) \chi_{0}(y)
$$

such that,

$$
a^{A} \chi_{0}(y)=0, \quad \int d y \chi_{0}^{2}(y)=1 .
$$

The expectation value of $H$ up to first order in $\hbar$ is given by

$$
\left\langle\psi_{0}|H| \psi_{0}\right\rangle=\int d x \sqrt{G} T^{*}(x)\left\langle\left({ }^{\epsilon} \mathscr{H}^{(0)}+\hbar^{\epsilon} \mathscr{H}^{(2)}\right)\right\rangle_{0}^{\perp} T(x),
$$


where we have used

$$
\langle\mathcal{O}\rangle_{0}^{\perp}=\int d y \chi_{0}(y) \mathcal{O} \chi_{0}(y) .
$$

Using the results

$$
\begin{gathered}
\left\langle\mathscr{H}^{(0)}\right\rangle_{0}^{\perp}=\frac{1}{2} \sum_{A} \epsilon_{A}, \\
\left\langle{ }^{\epsilon} \Lambda_{A B}\right\rangle_{0}^{\perp}=0, \\
\left\langle\Lambda^{\epsilon} \Lambda_{A B}^{\epsilon} \Lambda_{C D}\right\rangle_{0}^{\perp}=\frac{\left(\epsilon_{A}-\epsilon_{B}\right)^{2}}{16 \epsilon_{A} \epsilon_{B}}\left(\eta_{A C} \eta_{B D}-\eta_{A D} \eta_{B C}\right), \\
\left\langle y^{A} y^{C} y^{D} y^{B}\right\rangle_{0}^{\perp}=\frac{1}{4}\left(\eta^{C D} \eta^{A B}+\eta^{C B} \eta^{A D}+\eta^{A C} \eta^{D B}\right),
\end{gathered}
$$

one finds for the effective Hamiltonian $\mathscr{H}_{\text {eff }}$ for $T(x)$,

$$
\begin{gathered}
\left\langle\psi_{0}|H| \psi_{0}\right\rangle=\hbar \int d x \sqrt{G(x)} T^{*}(x) \mathscr{H}_{\mathrm{eff}} T(x), \\
\mathscr{H}_{\mathrm{eff}}=-\frac{1}{2} \nabla^{2}+\frac{m^{2}}{2}+V_{\mathrm{eff}}^{\bar{r}}(x)+V_{\mathrm{eff}}^{\bar{\omega}}(x),
\end{gathered}
$$

where

$$
\begin{gathered}
m^{2}=\frac{1}{\hbar} \sum_{A} \epsilon_{A}, \\
V_{\mathrm{eff}}^{\bar{r}}(x)=-\frac{1}{4} \bar{r}_{\|}-\frac{1}{12} \bar{r}_{\perp} \\
+\sum_{A, B}\left[\frac{\left(\epsilon_{A}-\epsilon_{B}\right)^{2}}{48 \epsilon_{A} \epsilon_{B}}-\frac{\left(\epsilon_{A}-\epsilon_{B}\right)}{24 \epsilon_{B}}\right] \bar{r}_{A B}^{A B}, \\
V_{\mathrm{eff}}^{\bar{\omega}}(x)=\sum_{B, D} \frac{\left(\epsilon_{B}-\epsilon_{D}\right)^{2}}{16 \epsilon_{B} \epsilon_{D}} \bar{\omega}^{\alpha B D} \bar{\omega}_{\alpha B D} .
\end{gathered}
$$

As the reason will be explained in Section 5, we identify the following equation as the analogue of linearized tachyon effective equation:

$$
\mathscr{H}_{\text {eff }} T(x)=0 .
$$

\section{Tubular Neighborhood of Target Manifold in Loop Space}

The leading order analysis of the finite dimensional model as done in Section 3 will be interpreted in the context of LSQM in Section 5. As motivated in Section 1, the submanifold of interest in this case is the space of all constant loops which is isomorphic to $\mathscr{M}$ itself (see Appendix C). The main ingredient that has gone into the analysis of Section 3 is the tubular expansion of the ambient space metric up to quadratic order as given in (3) with the additional condition (8). The goal of this section will be to understand the analogue of this expansion in $\mathscr{L} \mathscr{M}$. More precisely, we will show that (3) and (8) will still be valid, with the notations correctly interpreted, for the embedding $\mathscr{M} \hookrightarrow \mathscr{L} \mathscr{M}$ with suitably constructed FNC and with additional equations, given in (48), that relate the relevant expansion coefficients to intrinsic geometric data of $\mathscr{M}$.

The steps that we will follow are as follows. First we present an explicit construction of the tubular neighborhood in Section 4.1. A proof of existence was given through an explicit construction earlier by Stacey in [29]. Although the basic ideas are similar, the details of our construction are different and have been chosen to suit our purpose (in Section 4.2) better. The result of Section 4.1 will show how the relevant Riemannian structure in $\mathscr{L} \mathscr{M}$ is related to a certain Riemannian structure on the tangent bundle $T \mathscr{M}$ of $\mathscr{M}$. The latter views the zero-section $T \mathscr{M}_{0}$ (which is isomorphic to $\mathscr{M})$ as a submanifold embedded in $T \mathscr{M}$ such that the normal bundle $N\left(T \mathscr{M}_{0}\right)$ is isomorphic to $T \mathscr{M}$ itself. In Section 4.2 we first compute the tubular expansion of the metric in $T \mathscr{M}$ up to quadratic order using a procedure that is implicit in the construction of Section 4.1. The expansion coefficients are all related to intrinsic geometric data of $\mathscr{M}$ (For any given Riemannian structure on $T \mathscr{M}$, one would expect that the geometric data of $T \mathscr{M}$ should be expressible in terms of those of $\mathscr{M}$. See, e.g., [33].). Such relations have been found up to an undetermined real parameter which is not fixed at the present level of approximation by the analogue of (A.2) which determined the tubular expansion of vielbein in the finite dimensional context. Finally, we derive the metric expansion in $\mathscr{L} \mathscr{M}$ (up to quadratic order) using the aforementioned relation between $\mathscr{L} \mathscr{M}$ and $T \mathscr{M}$. Therefore, the ambiguity of the real parameter mentioned above is carried over to $\mathscr{L} \mathscr{M}$. However, as explained in Section 5, this ambiguity does not affect our conclusion about the tachyon effective equation.

4.1. Explicit Construction of the Tubular Neighborhood. Below we will first heuristically describe our construction and then specify it in more mathematical terms, in particular make connection with [29]. We will refer to geodesics and open neighborhoods in both $\mathscr{L} \mathscr{M}$ and $\mathscr{M}$ in various places along the way. It should be clear from the context which space we are referring to.

The basic picture [17], true for any tubular neighborhood, that we will have in mind is given in Figure 1. Given any point $Q$ in the neighborhood, there exists a unique geodesic passing through $Q$ that arrives at a unique point $P$ on the submanifold orthogonally.

This condition is not satisfied if two geodesics emerging orthogonally from the submanifold meet at a point outside. Following the standard way, we will restrict ourselves to a region sufficiently close to the submanifold such that this does not happen. Recall that every point in the neighborhood in $\mathscr{L} \mathscr{M}$ corresponds to a nonzero loop in $\mathscr{U}$ such that the nearer the point resides to the submanifold of vanishing loops, the smaller the loop it represents. It turns out that the above restriction corresponds to considering sufficiently small loops in $\mathscr{M}$ such that any given loop can be entirely encompassed within a single convex normal neighborhood [34] in $\mathscr{M}$. This implies that a small loop should fit entirely into $B_{p}$-the ball of largest RNC-radius with center at $p \in M$, for some $p$ in the neighborhood. 


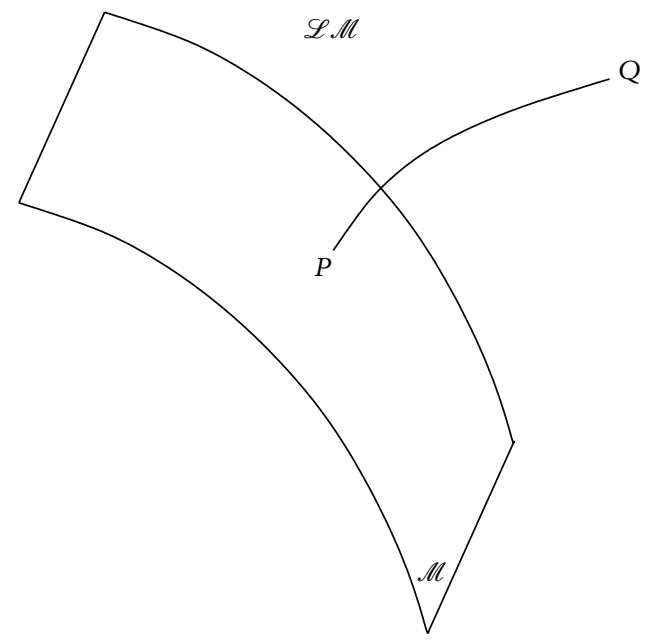

Figure 1: Tubular neighborhood of $\mathscr{M}$ in $\mathscr{L} \mathscr{M}$.

Let us now consider the set of points lying on the geodesic $Q P$ in $\mathscr{L} \mathscr{M}$. This corresponds to a class of loops which progressively shrink to zero size as we approach $P$ (see cartoon in Figure 2). Therefore, from the perspective of the interior of $\mathscr{M}$ this defines $P$ to be some kind of an average value for all the loops in this class. Notice that such a definition of averaging is independent of the choice of coordinate system, simply because it only refers to geodesics.

Given a loop-embedding $Z$ in $\mathscr{M}$, its average position, as defined above, can be found in the following way. In case $\mathscr{M}$ is flat, it is simply given by $\int_{S^{1}} Z$. This is basically because Minkowski space is also a vector space where one can define a radial vector. In a curved space one should make use of geodesics which look like radial vectors in RNC, the latter being related to general coordinates through the exponential map. Therefore, when $\mathscr{M}$ is curved, we first describe the loop in RNC centered at a suitable point, with coordinate say $x$. The choice of this base point is not fixed, as the loop will in general fit into $B_{x}$ for a range of values of $\underline{x}$. However, there is a unique value $x$ within the allowed range for which the following condition is satisfied:

$$
\int_{S^{1}} \widehat{Y}=0
$$

where $\widehat{Y}$ is the loop in RNC centered at $x$. Given the initial loop in $\mathscr{M}$, we identify $x$ as the average position. This way every small loop is uniquely assigned an average position in $\mathscr{M}$. Moreover, every point in a suitable neighborhood of $\mathscr{M}$ is uniquely assigned to a class of small loops through the above procedure which is viewed from the directions normal to $\mathscr{M}$ in $\mathscr{L} \mathscr{M}$.

To facilitate the discussion in next subsection we now describe the above construction in more technical terms. We begin by introducing the following notation: $U_{S}$ is open in $S$ where $S$ stands for $\mathscr{M}, \mathscr{M} \times \mathscr{M}, T \mathscr{M}$, and $\mathscr{L} \mathscr{M}$. We will see how these various open neighborhoods are interrelated. Given an open normal neighborhood $U_{\mathscr{M}} \subset \mathscr{M}$, let $C\left(U_{\mathscr{M}}\right)$ be the collection of all small loops such that the average positions

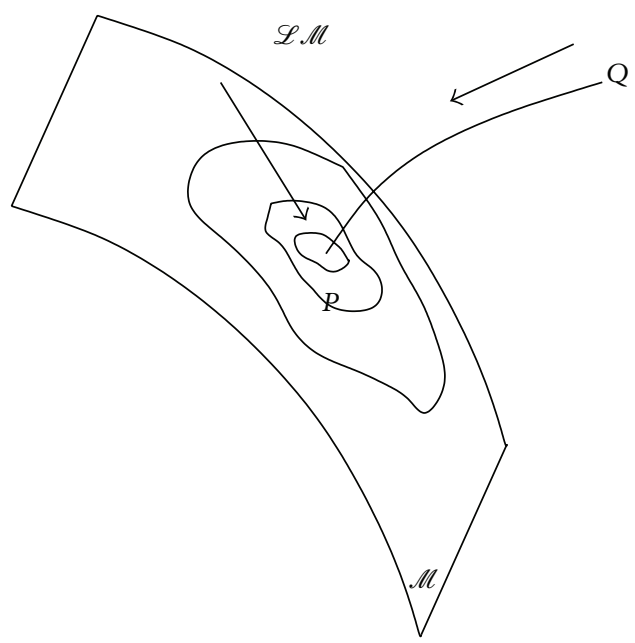

FIGURE 2: Average position of loops.

of all such loops are contained in $U_{\mathscr{M}}$. We also assume that all the loops in $C\left(U_{\mathscr{M}}\right)$ are contained in a single open normal neighborhood $\widetilde{U}_{\mathscr{M}} \supset U_{\mathscr{M}}$. Each element $l \in C\left(U_{\mathscr{M}}\right)$ can be associated with an element $(x, Z(\sigma)) \in U_{\mathscr{M} \times, \mathscr{M}}$ where $Z(\sigma)$ is the embedding of the loop $l$ given in general coordinates (see Appendix C), $x$ is the average position of the loop, and $U_{\mathscr{M} \times \mathscr{M}}$ is a suitable open neighborhood containing all the loops in $C\left(U_{\mathscr{M}}\right)$. The preimage of the loop under the exponential map found in $T_{x} \mathscr{M}$ is given by $\widehat{Y}(\sigma)$ which satisfies (25). Repeating this procedure for all the loops in $C\left(U_{\mathscr{M}}\right)$ one arrives at the following set [29]:

$$
U_{\mathscr{L} \mathscr{M}}=\left\{\widehat{Y}: S^{1} \longrightarrow U_{T \mathscr{M}}, \pi \widehat{Y} \text { is constant, } \int_{S^{1}} \widehat{Y}=0\right\},
$$

where $\pi: U_{T \mathscr{M}} \rightarrow U_{\mathscr{M}}$ is the projection map. Constancy of $\pi \widehat{Y}$ implies that the whole loop resides in the same fibre, unlike its configuration in $\mathscr{M} \times \mathscr{M}$.

Since exponential map is a diffeomorphism, the above argument shows that the desired tubular neighborhood is diffeomorphic to the set in (26). The relevant diffeomorphism is a bundle map which is the collection of all the inverse exponential maps at all $x \in U_{\mathscr{M}}$,

$$
\exp ^{-1}: U_{\mathscr{M} \times \mathscr{M}} \longrightarrow U_{T \mathscr{M}} .
$$

If $\Delta$ is the diagonal submanifold of $\mathscr{M} \times \mathscr{M}$, then $\exp ^{-1}$ maps $U_{\mathscr{M} \times \mathscr{M}} \cap \Delta$ to $U_{T \mathscr{M}} \cap T \mathscr{M}_{0}$, both being isomorphic to $U_{\mathscr{M}}$. This kind of a construction is called a local addition (see [35] for a precise definition) of which the exponential map is a standard example. In [29] construction of the local addition has been facilitated by embedding $\mathscr{M}$ in a higher dimensional Euclidean space. However, exponential map is more suitable for our purpose, as we will see in the next subsection where the aforementioned diffeomorphism will be explicitly constructed.

4.2. Construction of FNC. Here we would like to understand the loop space analogue of the metric expansion given in 
(3). As mentioned before, generically the tubular expansion coefficients are certain geometric quantities of the ambient manifold evaluated on the submanifold. Although these are not related to the intrinsic geometric properties of the submanifold in general, for loop space, that is, the case. Therefore, expressing the tubular expansion coefficients of $\mathscr{L} \mathscr{M}$ in terms of the geometric data of $\mathscr{M}$ is the precise quantitative question that needs to be answered. The discussion in the previous subsection implicitly defines a procedure to answer this question which we pursue here.

There are two steps to be followed. Given a Riemannian structure on $\mathscr{M}$, the space $\mathscr{M} \times \mathscr{M}$ acquires a natural direct product structure. The bundle map (diffeomorphism) in (27) enables one to view T $\mathscr{M}$ as a Riemannian manifold where $T \mathscr{M}_{0}$ sits as a submanifold whose normal bundle $N\left(T \mathscr{M}_{0}\right)$ is isomorphic to $T \mathscr{M}$. The first step is to construct the relevant submanifold based coordinate system, which we call $\mathrm{FNC}_{U_{T, \mu}}$, on $U_{T, \mathscr{M}}$ by a suitable coordinate transformation from the direct product coordinate system on $U_{\mathscr{M} \times \mathscr{M}}$. This will be discussed in Section 4.2.1. Then the final step is to construct FNC in $\mathscr{L} \mathscr{M}$ by looping $\mathrm{FNC}_{U_{T, M}}$, a procedure that has been explained in Section 4.2.2.

4.2.1. Riemannian Structure on T M. In Section 2 we considered a submanifold $(M, G)$ embedded in an ambient space $(L, g)$ such that $G$ is the induced metric obtained from $g$. Here we consider a special case where $\left(T \mathscr{M}_{0} \cong \mathscr{M}, G\right)$ is embedded in $(T \mathscr{M}, \widehat{g})$. The speciality of this case is that the tubular expansion coefficients are related to quantities obtained from the basic data $(\mathscr{M}, G)$ (Because of the involvement of spin connection, we will see that the basic data is actually given by the vielbein of $\mathscr{M}$.). Below we will construct $\mathrm{FNC}_{U_{T, \|}}$ up to quadratic order by starting with $(\mathscr{M} \times \mathscr{M}, \bar{g})$ and performing suitable coordinate transformations. There will be a certain degree of indeterminacy in our final result which, as will be explained toward the end of this subsection, is not resolved at the present level of approximation by the analogue of (A.2).

We begin by discussing $(\mathscr{M} \times \mathscr{M}, \bar{g})$. The coordinates of a point in $U_{\mathscr{M} \times \mathscr{M}}$ are given by

$$
\bar{z}^{a}=\left(x_{1}^{\alpha_{1}}, x_{2}^{\alpha_{2}}\right), \quad \alpha_{1}, \alpha_{2}=1, \ldots, D(=\operatorname{dim} \mathscr{M}) .
$$

The components of the vielbein are given by

$$
\bar{e}_{b}^{\left[\alpha_{1}\right]}(\bar{z})=\left(\begin{array}{c}
E^{\left(\alpha_{1}\right)} \beta_{1}\left(x_{1}\right) \\
0
\end{array}\right), \quad \bar{e}_{b}^{\left[\alpha_{2}\right]}(\bar{z})=\left(\begin{array}{c}
0 \\
E_{\beta_{2}}^{\left(\alpha_{2}\right)}\left(x_{2}\right)
\end{array}\right) .
$$

Indices in square brackets refer to the noncoordinate basis in $\mathscr{M} \times \mathscr{M}$. $E^{(\alpha)}{ }_{\beta}$ are the vielbein components of $\mathscr{M}$ (with indices in round brackets referring to the noncoordinate basis) with metric components given by $G_{\alpha \beta}$

$$
G_{\alpha \beta}=E_{\alpha}^{(\gamma)} E_{(\gamma) \beta} .
$$

In general the two copies of $\mathscr{M}$ can have different metrics which are diffeomorphic to each other. We have chosen $x_{1}$ and $x_{2}$ suitably so that these two metrics are the same as given in (30). We will denote the desired coordinate system
FNC $_{U_{T, \text { II }}}$ by $\widehat{z}=\left(x^{\alpha}, \widehat{y}^{\widehat{\alpha}}\right)$ with $\alpha, \widehat{\alpha}=1, \ldots, D$ (Notice that the indices $\alpha_{1}, \alpha_{2}, \alpha$, and $\widehat{\alpha}$ all run over $D=\operatorname{dim} \mathscr{M}$ dimensions. From the perspective of $\mathscr{M}$ they do not make any difference. However, from the perspective of $\mathscr{M} \times \mathscr{M}$ they do.), which will be obtained below by following a series of coordinate transformations from $\bar{z}$.

The first step is to argue, as has been done in Appendix D, that there exists a coordinate system

$$
z^{\prime a}=\left(x^{\prime \alpha}, y^{\prime \widehat{\alpha}}\right)
$$

where $x^{\prime}$ is a general coordinate system on $\Delta_{U_{\mathscr{M} \times M}}=U_{\mathscr{M} \times \mathscr{M}} \cap$ $\Delta$ such that the transformed components of the vielbein (with an additional overall constant scaling of the metric, see Appendix D) are given by the following expansion up to quadratic order in $y^{\prime}$

$$
\begin{aligned}
& e_{\beta}^{\prime[\alpha]}\left(z^{\prime}\right)=E_{\beta}^{(\alpha)}\left(x^{\prime}\right)+q \check{R}^{(\alpha)}{ }_{\hat{\gamma} \hat{\beta}}\left(x^{\prime}\right) y^{\prime \hat{\gamma}} y^{\prime \widehat{\delta}}, \\
& e^{\prime[\alpha]}{ }_{\widehat{\beta}}\left(z^{\prime}\right)=0, \\
& e_{\beta}^{\prime[\widehat{\alpha}]}\left(z^{\prime}\right)=0, \\
& e^{\prime[\widehat{\alpha}]}{ }_{\widehat{\beta}}\left(z^{\prime}\right)=E_{\hat{\beta}}^{(\widehat{\alpha})}\left(x^{\prime}\right)+\frac{1}{6} \check{R}_{\hat{\gamma} \widehat{\gamma} \hat{\beta}}^{(\widehat{\alpha})}\left(x^{\prime}\right) y^{\prime \widehat{\gamma}} y^{\prime \widehat{\delta}} \text {, }
\end{aligned}
$$

where the orthonormal frames with superscripts $[\alpha]$ and $[\widehat{\alpha}]$ are parallel and transverse to the submanifold, respectively, and are obtained from the ones in (29) through a rotation of the local Lorentz frame as given in (D.5). The symbol $\check{R}_{\gamma \delta \beta}^{(\alpha)}\left(x^{\prime}\right)$ denotes the Riemann curvature tensor component $R^{(\alpha)}{ }_{\gamma \delta \beta}$ (we use upper case symbols without a ${ }^{2}$ to denote tensors of $\mathscr{M}$ in general coordinates) of $\mathscr{M}$ evaluated at $x^{\prime}$ in $\mathrm{RNC}_{x^{\prime}}$ where $\mathrm{RNC}_{x^{\prime}}$ refers to the RNC-system centered at $x^{\prime}$ such that the vielbein components are given by $E^{(\alpha)}{ }_{\beta}\left(x^{\prime}\right)$ at the centre. $q$ is an undetermined real number. We will argue toward the end of this subsection that the analogue of (A.2) is satisfied up to quadratic order for arbitrary values of $q$.

Then the final step is to perform the following coordinate transformation: $z^{\prime a} \rightarrow \widehat{z}^{a}=\left(x^{\alpha}, \widehat{y}^{\widehat{\alpha}}\right)$ such that

$$
x^{\alpha}=x^{\prime \alpha}, \quad \widehat{y}^{\widehat{\alpha}}=E_{\widehat{\beta}}^{(\widehat{\alpha})}(x) y^{\prime \widehat{\beta}} .
$$

The transformed vielbein components are given by

$$
\begin{aligned}
& \hat{e}_{\beta}^{[\alpha]}(\widehat{z})=E_{\beta}^{(\alpha)}(x)+q \check{R}^{(\alpha)}{ }_{\hat{\gamma} \hat{\delta} \beta}(x) \hat{y}^{\widehat{\gamma}} \widehat{y}^{\widehat{\delta}}, \\
& \widehat{e}^{[\alpha]}{ }_{\widehat{\beta}}(\widehat{z})=0, \\
& \widehat{e}_{\beta}^{[\widehat{\alpha}]}(\widehat{z})=E^{(\widehat{\alpha})}{ }_{\widehat{\delta}}(x) \partial_{\beta} E_{(\widehat{\gamma})}{ }^{\widehat{\delta}}(x) \hat{y}^{\widehat{y}}, \\
& \hat{e}^{[\hat{\alpha}]}{ }_{\hat{\beta}}(\widehat{z})=\delta_{\hat{\beta}}^{\widehat{\alpha}}+\frac{1}{6} \check{R}_{(\widehat{\gamma} \hat{\delta} \widehat{\beta})}^{(\hat{\alpha})}(x) \hat{y}^{\widehat{\gamma}} \hat{y}^{\widehat{\delta}},
\end{aligned}
$$


which give the following results for the metric components,

$$
\begin{aligned}
& \widehat{g}_{\alpha \beta}=G_{\alpha \beta}+\overline{\widehat{s}}_{\alpha \beta \widehat{\gamma}} \widehat{y}^{\hat{\gamma}} \\
& +\left(\overline{\widehat{\omega}}_{\alpha}^{\eta} \overline{\widehat{\omega}}_{\beta \eta \widehat{\delta}}+\overline{\widehat{\omega}}_{\alpha}^{\hat{\eta}} \overline{\widehat{\omega}}_{\beta \hat{\eta} \delta}+\overline{\widehat{r}}_{\alpha \hat{\gamma} \hat{\delta} \beta}\right) \widehat{y}^{\widehat{\gamma}} \widehat{y}^{\widehat{\delta}} \\
& +O\left(\hat{y}^{3}\right) \text {, } \\
& \widehat{g}_{\alpha \hat{\beta}}=\overline{\widehat{\omega}}_{\alpha \widehat{\beta} \hat{\gamma}} \widehat{y}^{\widehat{\gamma}}+\frac{2}{3} \overline{\widehat{r}}_{\alpha \widehat{\gamma} \delta \hat{\beta}} \hat{y}^{\hat{\gamma}} \widehat{y}^{\widehat{\delta}}+O\left(\hat{y}^{3}\right), \\
& \widehat{g}_{\hat{\alpha} \hat{\beta}}=\eta_{\widehat{\alpha} \hat{\beta}}+\frac{1}{3} \overline{\hat{r}}_{\widehat{\alpha} \hat{\gamma} \delta \hat{\beta} \hat{\gamma}} \widehat{y}^{\hat{\gamma}} \widehat{y}^{\widehat{\delta}}+O\left(\hat{y}^{3}\right),
\end{aligned}
$$

with

$$
\begin{gathered}
\overline{\widehat{\omega}}_{\alpha \beta \widehat{\gamma}}=0, \quad\left(\Longrightarrow \overline{\hat{s}}_{\alpha \beta \widehat{\gamma}}=0\right), \\
\overline{\widehat{\omega}}_{\alpha \widehat{\beta} \widehat{\gamma}}=E_{(\widehat{\beta}) \widehat{\delta}}(x) \partial_{\alpha} E_{(\widehat{\gamma})} \widehat{\delta}(x), \\
\overline{\widehat{\hat{r}}}_{\alpha \widehat{\gamma} \hat{\delta} \beta}=2 q \check{R}_{\alpha(\widehat{\gamma} \hat{\delta}) \beta}(x), \\
\overline{\widehat{\hat{r}}}_{\alpha \widehat{\gamma} \hat{\delta} \hat{\beta}}=0, \\
\overline{\widehat{r}}_{\widehat{\alpha} \hat{\gamma} \hat{\delta} \hat{\beta}}=\check{R}_{(\widehat{\alpha} \hat{\gamma} \hat{\delta} \widehat{\beta})}(x) .
\end{gathered}
$$

A few remarks about (34), (35), and (36) are in order. All the hatted variables appearing in these equations are tensors of $\mathscr{M} \times \mathscr{M}$ in $\widehat{z}=(x, \hat{y})$ coordinate system. Equivalently, they can also be viewed as tensors of $T \mathscr{M}$. In particular, (34) and (35) are viewed to describe the tubular expansion of vielbein and metric components, respectively, up to quadratic order in FNC. Notice that (35) are written in the general form of tubular expansion as in (3) following the same rules for notation adopted there. Therefore a bar indicates that a tensor of $T \mathscr{M}$ is being evaluated on $U_{T, \mathscr{M}} \cap T \mathscr{M}_{0}$. Equations (36) exhibit how such quantities are related to intrinsic geometric quantities of $\mathscr{M}$. Finally, the results in (35) satisfy the analogue of the coordinate conditions in (2) because of the antisymmetry properties of the spin connection and curvature tensor identified in (36) (Notice that the spin connection identified in the second equation of (36) is indeed antisymmetric in the last two indices as required.).

The tubular expansion in (34) is supposed to satisfy the analogue of the differential equation (A.2) in T.M. This is given, in our notation adopted here, by

$$
\widehat{\mathbf{d}}\left(\widehat{\mathbf{d}}+\stackrel{b}{\epsilon}^{b}\right) \widehat{e}^{[a]}{ }_{b}(\widehat{z})=\hat{\rho}_{[c]}^{[a]}(\widehat{z} ; \widehat{y}) \hat{e}^{[c]}{ }_{b}(\widehat{z}),
$$

where $\widehat{\mathbf{d}}=\widehat{y}^{\widehat{\alpha}}\left(\partial / \partial \widehat{y}^{\widehat{\alpha}}\right), a=\alpha, \widehat{\alpha}, \epsilon^{b}$ being 1 for $b=\widehat{\beta}$ and -1 , otherwise and

$$
\hat{\rho}_{[b]}^{[a]}\left(\widehat{z} ; \widehat{y}^{\prime}\right)=\widehat{r}^{[a]}{ }_{\hat{\gamma} \widehat{\delta}[b]}(\widehat{z}) \hat{y}^{\prime \hat{\gamma}} \hat{y}^{\widehat{\delta}} .
$$

It is straightforward to to check that (37) is satisfied by (34) up to quadratic order for arbitrary values of $q$ provided that the curvature components of $T \mathscr{M}$ are identified according to the last three equations in (36).
4.2.2. Looping $F N C_{T, \mathscr{M}}$. The desired FNC in $\mathscr{L} \mathscr{M}$ is obtained by looping the coordinate system $\widehat{z}^{\widehat{a}}=\left(x^{\alpha}, \widehat{y}^{\widehat{\alpha}}\right)$ constructed in the previous subsection. To explain the method we first recall the following facts.

(1) The normal bundle $N\left(T \mathscr{M}_{0} \cong \mathscr{M}\right)$ is isomorphic to $T \mathscr{M} \cdot \hat{y}^{\widehat{\alpha}}$ are the coordinates on the fibres $N_{x}\left(T \mathscr{M}_{0}\right)$.

(2) The desired tubular neighborhood in $\mathscr{L} \mathscr{M}$ is given by the space of nonzero loops in (26). Here every loop resides entirely in a single fibre $T_{x}(\mathscr{M})$ such that the average of the loop is the corresponding base point $x$.

Therefore, the general coordinate $x^{\alpha}$ on the submanifold $\mathscr{M} \hookrightarrow \mathscr{L} \mathscr{M}$ is the same as that on $T \mathscr{M}_{0} \hookrightarrow T \mathscr{M}$. And the normal coordinate in the neighborhood of $\mathscr{M} \hookrightarrow \mathscr{L} \mathscr{M}$ is given by the Fourier transforms (see Appendix C) of the loop in $T_{x}(\mathscr{M})$ as described above. In terms of equations, the FNC in $\mathscr{L} \mathscr{M}$ is given by

$$
z^{a}=\left(x^{\alpha}, y^{A}\right)
$$

where

$$
y^{A}=\oint \widehat{Y}^{\widehat{\alpha}}(\sigma) e^{-i \mathrm{a} \sigma}, \quad \mathrm{a} \neq 0,
$$

such that $\widehat{Y}^{\widehat{\alpha}}(\sigma)$ satisfies (25).

Tensors in $\mathscr{L} \mathscr{M}$ are obtained from those in $T \mathscr{M}$ following a similar procedure $[14,15]$ which we discuss in detail now. Let $\left\{\widehat{e}_{\widehat{a}}\right\}=\left\{\widehat{e}_{\alpha}=\partial / \partial x^{\alpha}, \widehat{e}_{\widehat{\alpha}}=\partial / \partial \widehat{y}^{\widehat{\alpha}}\right\}$ and $\left\{d \widehat{z}^{\widehat{a}}\right\}=$ $\left\{d x^{\alpha}, d \widehat{y}^{\widehat{\alpha}}\right\}$ be the coordinate basis (in special coordinate system constructed in previous subsection) for the tangent and cotangent spaces $T_{\bar{z}}(T \mathscr{M})$ and $T_{\bar{z}}^{*}(T \mathscr{M})$, respectively, at $\widehat{z}$ in $U_{T, M}$. A rank $(m, n)$ tensor is given by

$$
\widehat{t}=\widehat{t}^{\widehat{a}_{1} \cdots \widehat{a}_{m}} \widehat{b}_{1} \cdots \hat{b}_{n}(x, \hat{y}) \widehat{e}_{\widehat{a}_{1}} \cdots \widehat{e}_{\widehat{a}_{m}} d \widehat{z}^{\widehat{b}_{1}} \cdots d \widehat{z}^{\widehat{b}_{n}} .
$$

The tubular expansion of the components takes the following form:

$$
\widehat{t}^{\hat{a}_{1} \cdots \widehat{a}_{m}} \widehat{b}_{1} \cdots \widehat{b}_{n}(x, \hat{y})=\sum_{p \geq 0} \overline{\hat{t}}_{p}^{\widehat{a}_{1} \cdots \widehat{a}_{m}} \widehat{b}_{1} \cdots \hat{b}_{n} \widehat{\delta}_{1} \cdots \widehat{\delta}_{p}(x) \widehat{y}^{\widehat{\delta}_{1}} \cdots \widehat{y}^{\widehat{\delta}_{p}},
$$

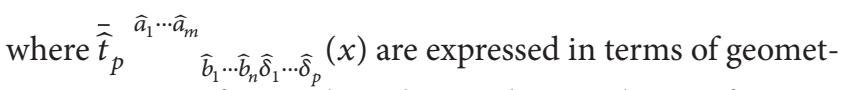
ric quantities of $\mathscr{M}$ evaluated at $x$. The coordinate of a point in $U_{\mathscr{L} \mathscr{M}}$ is given by (39). The coordinate basis for the tangent and cotangent spaces $T_{z}(\mathscr{L} \mathscr{M})$ and $T_{z}^{*}(\mathscr{L} \mathscr{M})$ are given by $\left\{e_{a}\right\}=\left\{e_{\alpha}=\partial / \partial x^{\alpha}, e_{A}\right\}$ and $\left\{d z^{a}\right\}=\left\{d x^{\alpha}, d y^{A}\right\}$, respectively, where

$$
e_{A}=\oint e^{i a \sigma} \frac{\delta}{\delta \widehat{Y}^{\hat{\alpha}}(\sigma)}, \quad d y^{A}=\oint e^{-i a \sigma} \delta \widehat{Y}^{\hat{\alpha}}(\sigma),
$$

$\delta \widehat{Y}^{\widehat{\alpha}}(\sigma)$ being a functional differential. The tensor corresponding to that in (41) is given by (Definition (44), (45) is equivalent to the following alternative expression, 


$$
\begin{aligned}
& t=\oint t^{\widehat{a}_{1} \cdots \widehat{a}_{m}} \widehat{b}_{b_{1} \cdots \bar{b}_{n}}(x, Y(\sigma))\left(\delta / \delta \widehat{z}^{\widehat{a}_{1}}(\sigma)\right) \cdots\left(\delta / \delta \widehat{z}^{\widehat{a}_{m}}(\sigma)\right) d \widehat{z}^{\widehat{b}_{1}}(\sigma) \\
& \left.\cdots d \bar{z}^{b_{n}}(\sigma) \text {, where } \widehat{z}^{\widehat{a}}(\sigma)=\left(x^{\alpha}, \widehat{Y}^{\widehat{\alpha}}(\sigma)\right) .\right) \\
& \qquad t=t_{b_{1} \cdots b_{n}}^{a_{1} \cdots a_{a}}(x, y) e_{a_{1}} \cdots e_{a_{m}} d z^{b_{1}} \cdots d z^{b_{n}}, \quad(44)
\end{aligned}
$$

where

$$
\begin{aligned}
& t^{a_{1} \cdots a_{m}} b_{1} \cdots b_{n} \\
& \quad=\oint \widehat{t}^{\widehat{a}_{1} \cdots \widehat{a}_{m}} \widehat{b}_{1} \cdots \widehat{b}_{n} \\
&
\end{aligned}
$$

Similar expression holds for the tubular expansion

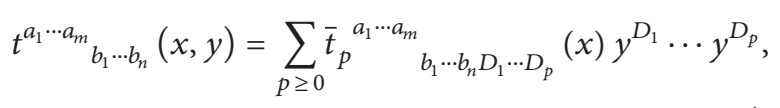

where

$$
\begin{aligned}
\bar{t}_{p}^{a_{1} \cdots a_{m}} & b_{1} \cdots b_{n} D_{1} \cdots D_{p} \\
= & \oint \overline{\hat{t}}_{p}^{\widehat{a}_{1} \cdots \widehat{a}_{m}} \widehat{b}_{1} \cdots \hat{b}_{n} \widehat{\delta}_{1} \cdots \widehat{\delta}_{p}(x) \\
& \times e^{-i\left(a_{1}+\cdots+a_{m}\right) \sigma+i\left(\mathrm{~b}_{1}+\cdots+b_{n}+\mathrm{d}_{1}+\cdots+\mathrm{d}_{p}\right) \sigma} \\
= & \overline{\widehat{t}}_{p}^{\widehat{a}_{1} \cdots \widehat{a}_{m}} \hat{b}_{1} \cdots \hat{b}_{n} \widehat{\delta}_{1} \cdots \widehat{\delta}_{p}(x) \\
& \times \delta_{-\mathrm{a}_{1}-\cdots-\mathrm{a}_{m},+\mathrm{b}_{1}+\cdots+\mathrm{b}_{n}+\mathrm{d}_{1}+\cdots+\mathrm{d}_{p}, 0 .} .
\end{aligned}
$$

Notice that the notations adopted in this subsection for the FNC and tensors of $\mathscr{L} \mathscr{M}$ are the same as those for the FNC and tensors of $L$ considered in Section 2. This makes the expressions for the tubular expansion of various tensors of $\mathscr{L} \mathscr{M}$ look exactly the same as the corresponding general expressions in the finite dimensional case with the only additional input that the expansion coefficients are related to certain geometric data of $\mathscr{M}$. Such relations are inherited from their counterparts in $T \mathscr{M}$ through the above framework which relates tensors of $\mathscr{L} \mathscr{M}$ to those of $T \mathscr{M}$. In particular, one finds that the expansions of the loop-spacemetric-components are given by the same equations as in (3) with the following identifications:

$$
\begin{gathered}
\eta_{A B}=\eta_{(\widehat{\alpha} \widehat{\beta})} \delta_{\mathrm{a}+\mathrm{b}, 0}, \\
\bar{\omega}_{\alpha \beta D}=0, \quad\left(\Longrightarrow \bar{s}_{\alpha \beta D}=0\right), \\
\bar{\omega}_{\alpha B D}=E_{(\widehat{\beta} \hat{\gamma} \hat{\gamma}}(x) \partial_{\alpha} E_{(\widehat{\delta})} \hat{\gamma}(x) \delta_{\mathrm{b}+\mathrm{d}, 0}, \\
\bar{r}_{\alpha D E \beta}=2 q \check{R}_{\alpha(\hat{\delta} \hat{\eta}) \beta}(x) \delta_{\mathrm{d}+\mathrm{e}, 0}, \\
\bar{r}_{\alpha B D E}=0, \\
\bar{r}_{A B D E}=\check{R}_{(\widehat{\alpha} \widehat{\beta} \widehat{\delta} \hat{\eta})}(x) \delta_{\mathrm{a}+\mathrm{b}+\mathrm{d}+\mathrm{e}, 0} .
\end{gathered}
$$

In particular, the above shows that $\mathscr{M} \hookrightarrow \mathscr{L} \mathscr{M}$ is a totally geodesic submanifold. See also comments below (C.6). With this we end our discussion of the explicit construction of the tubular neighborhood of $\mathscr{M} \hookrightarrow \mathscr{L} \mathscr{M}$ and the relevant FNC.

\section{Analogy with Finite Dimensional Model}

In Section 3 we discussed a finite-dimensional analogue of LSQM. The primary goal of this analysis was to work out the relevant details (that one would eventually like to understand for LSQM) in a finite-dimensional set up which is free of divergences. Here we will spell out how precisely to interpret the analysis of Section 3 in the context of string theory. Our final goal will be to understand the features of the expected tachyon effective equation, the analogue of which is described by (22), (23), and (24).

(i) The ambient space $L$ of the toy model is the configuration space and therefore is considered to be of Euclidean signature in Section 3. $\mathscr{M}$, on the other hand, is the extended configuration space (which includes time) of NLSM. In the context of LSQM, the analysis of Section 3 should be viewed as a worldline type theory. This has the following consequences.

(a) The theory is supplemented with the standard ghost sector of bosonic string theory. When $\mathscr{M}$ is taken to be pseudo-Riemannian, the potential $V$ (to be discussed further below) of the model will be maximized, instead of being minimized, on the submanifold along the time-like directions. This gives rise to the standard problem of negative norm states which is cured by the presence of ghost sector. With this understanding we will simply ignore this problem now onwards and assume $\mathscr{M}$ to have Euclidean signature.

(b) Hamiltonian is a constraint. The effective form of this constraint on the submanifold obtained by integrating out the transverse (internal) degrees of freedom is supposed to give the linearized equation of motion for the string field components on $\mathscr{M}$. This explains why (24) has been interpreted to be analogue of the linearized tachyon effective equation.

(ii) In the context of finite dimension in Section 2 we followed certain notation and convention for coordinate indices, FNC, tensors, and their tubular expansion (see first few paragraphs of Section 2). We followed the same rules in the context of $\mathscr{L} \mathscr{M}$ in Section 4.2.2. The prescription for translating any finite dimensional expression involving tubular expansion is simply to interpret the transverse indices (i.e., capital Latin indices) according to rules of loop space as described below (C.2) and evaluate the barred quantities involved in terms of the intrinsic data of $\mathscr{M}$ following (48).

(iii) We now explain the potential of the toy model. Equation (8) is simply the second equation in (48). This implies that the submanifold is totally geodesic (In the context of $\mathscr{L} \mathscr{M}$, as pointed out below (C.6), this is also related to the fact that the submanifold is the fixed-point set of the reparametrization Killing vector $v(z)$ in (C.3). Such a feature, however, is not 
shared by the toy model.). The relevance of (9) may be understood as follows. The potential $V^{\mathscr{L} \mathscr{M}}$ of LSQM, given in (C.7), can be written in terms of FNC as follows:

$$
\begin{aligned}
V^{\mathscr{L} \mathscr{M}} & =\frac{1}{2} \sum_{\mathrm{a}, \mathrm{b}}(-) \mathrm{ab} g_{A B} y^{A} y^{B} \\
& =\frac{1}{2} \sum_{\mathrm{a}, \mathrm{b}}\left(-\mathrm{ab} \eta_{A B} y^{A} y^{B}-\frac{1}{3} \mathrm{ab} \bar{r}_{A C D B} y^{A} y^{B} y^{C} y^{D}+\cdots\right),
\end{aligned}
$$

where in the second line we have used the metricexpansion up to quadratic order from (3). We perform the similar expansion in (9) and then compare with the loop space potential in (49) up to quartic order. At the quadratic order one finds, using the first equation in (48),

$$
\epsilon_{A}=|\mathrm{a}|
$$

With this identification, however, the terms at the quartic order fail to be equal as the relevant term in $V^{\mathscr{L} \mathscr{M}}$ is sensitive to the sign of the integer-prefactor ab. This changes the coefficient of the last term of $V_{\mathrm{eff}}^{\bar{r}}(x)$ in (23) (Note that rescaling of LSQM is defined by the same equations (10), (11) with the identification (50).). We will see that this does not affect our final conclusion about the tachyon effective equation.

Finally, unlike the toy model, the loop space potential $V^{\mathscr{L} \mathscr{M}}$ is invariant under the full GCT (see Appendix C) of $\mathscr{L} \mathscr{M}$. This happens because of the special property of loop space that it admits a vector field, that is, linear in coordinate. Therefore, the general covariance of LSQM is broken down to that of the submanifold only in the semiclassical vacuum, in particular, because of the rescaling (11).

(iv) The leading order rescaled Hamiltonian given by the first equation in (14) is analogous to the nonzero mode contribution to the Hamiltonian in flat space. The resemblance can be made more explicit through the following redefinition:

$$
\begin{array}{ll}
a^{A} \longrightarrow \alpha^{A}, & a^{\dagger \bar{A}} \longrightarrow \alpha^{\bar{A}}, \\
a^{\bar{A}} \longrightarrow \tilde{\alpha}^{A}, & a^{\dagger A} \longrightarrow \widetilde{\alpha}^{\bar{A}},
\end{array}
$$

where $\alpha$ and $\tilde{\alpha}$ are the usual flat-space-oscillators [36]. The index $\bar{A} \rightarrow(\alpha,-\mathrm{a})$ (see discussion below (C.2)) corresponds to a negative mode while $A$ to a positive mode. The leading order Hamiltonian takes the following familiar form in this new notation:

${ }^{\epsilon} \mathscr{H}^{(0)}=\sum_{\mathrm{a}, \mathrm{b}>0} \sqrt{\mathrm{ab}} \eta_{\bar{A} B}\left(\alpha^{\bar{A}} \alpha^{B}+\widetilde{\alpha}^{\bar{A}} \tilde{\alpha}^{B}\right)+\sum_{\mathrm{a}>0} \mathrm{a}$.

The last term is the zero-point energy which is divergent. Noticing that at the leading order the transverse dynamics exactly matches with that of the nonzero modes in flat space, this term can be treated in the usual manner. The point to be emphasized here is that in flat space such a term (after collecting the ghost contribution) finally gets related to the tachyon mass. The same is true here as we see from the first equation in (23). Notice also that this mass has the right scaling with respect to $\hbar=\alpha^{\prime}$. In fact, demanding that the leading order transverse Hamiltonian in (14) be precisely the same as the nonzero modes contribution to the Hamiltonian in flat space fixes the rescaling of the model as described by (10) and (11). Such a condition is required to get the right flat space limit (where the tubular expansion becomes trivial) of our analysis.

(v) Notice that while reinterpreting the oscillators of the toy model in terms of string modes through (51), the creation and annihilation operators do not mix up. This implies that the results for the vacuum expectation values in (21) are also valid in the context of LSQM. This enables us to view (22), (23), and (24) as describing the linearized tachyon effective equation at leading order in LSQM. One expects that such an equation should be given by

$$
\left(-\frac{1}{2} \nabla^{2}+\frac{m^{2}}{2}\right) T(x)=0,
$$

up to leading order equation of motion (i.e., Ricci flatness) for the background

$$
R_{\alpha \beta}(x)=0 .
$$

This equation has not been derived in this work as the toy model does not have an analogue of conformal invariance. However, one can imagine deriving this condition at the leading order of tubular expansion of the DeWitt-Virasoro algebra computed in $[14,15]$. We postpone this analysis for a future work and assume this is true for the time being.

We will now argue that

$$
\begin{gathered}
V_{\mathrm{eff}}^{\bar{r}}(x) \propto R(x), \\
V_{\mathrm{eff}}^{\bar{\omega}}(x)=0,
\end{gathered}
$$

where the proportionality constant in the first equation is divergent and this is the only divergence in the effective equation of motion at leading order. This is established simply by using the relevant equations in (48). Notice that the undetermined factor $q$ does not influence our final conclusion which is insensitive to the proportionality constant (it is divergent anyway). To establish the second equation one notices from the third equation in (48) that $\bar{\omega}_{\alpha B D}$ is nonzero only when $\mathrm{b}+\mathrm{d}=0$, in which case $\epsilon_{B}=\epsilon_{D}$ (according to the identification in (50)), and therefore $V_{\mathrm{eff}}^{\bar{\omega}}(x)$ in (23) vanishes. 
With this we end our discussion of how the computations in the finite-dimensional model discussed in Section 3 should be interpreted in the context of LSQM.

\section{Conclusion}

This work investigates how to make sense of a semiclassical limit of LSQM as discussed in [14, 15]. In this limit the wavefunction gets localized on the submanifold $\mathscr{M}$ of vanishing loops in $\mathscr{L} \mathscr{M}$ where $\mathscr{M}$ is the target space of the corresponding NLSM. The study involves first defining the procedure in a finite dimensional toy model (content of Sections 2 and 3) and then figuring out how the actual loop space model can be understood through an analogy with the toy model (content of Sections 4 and 5). The study shows that the linearized effective equation for the tachyon fluctuation at leading order in $\alpha^{\prime}$-expansion is reproduced correctly with all the divergent terms being proportional to the Ricci scalar of $\mathscr{M}$.

The present approach makes the usual picture of particle quantum mechanics quite explicit and therefore it is conceptually appealing. Given this, it is perhaps a good idea to work out the details of how the standard questions, such as Ricci-flatness as leading order condition for conformal invariance, low-energy effective equations of motion, and, most importantly, higher order $\alpha^{\prime}$ corrections, should be understood in the current approach. We hope that the analysis of the present work will be helpful for further study along this direction and its supersymmetrization.

We will conclude with a few remarks regarding the mathematical framework of Sections 4.1 and 4.2 where a certain Riemannian structure on $T \mathscr{M}$ was discussed. A speciality of this Riemannian structure is that it views $T \mathscr{M}_{0} \hookrightarrow T \mathscr{M}$ as a submanifold admitting a tubular neighborhood. Recall that an all-order understanding of tubular expansion of vielbein in a generic case is available through [19] (reviewed in Appendix A). This implies that finding the desired Riemannian structure on $T \mathscr{M}$ is equivalent to finding all the tubular expansion coefficients in terms of intrinsic geometric data of $\mathscr{M}$. In this work this has been done in a limited sense which proved sufficient for the present level of analysis of LSQM. It is possible that a more complete understanding of this question will be required for computing $\alpha^{\prime}$ corrections. We hope to come back to these questions in future.

Finally, we note that the mathematical framework discussed in Section 4.1 should also be relevant for a multiparticle classical system in curved space. The center of mass (CM) of the system belongs to the first copy of $\mathscr{M}$ in $\mathscr{M} \times \mathscr{M}$ while the positions relative to $\mathrm{CM}$ lie in the second copy. After performing the bundle map in (27) the CM resides in $T \mathscr{M}_{0}$ while the relative coordinates all lie on the same fibre whose base point is identified with the position of CM. In the limit when the potential is strong enough to hold all particles in the form of a rigid body, the dynamics confines on the submanifold $T \mathscr{M}_{0} \hookrightarrow T \mathscr{M}$. When we are away from this limit, the internal fluctuations are described in the tubular neighborhood of this embedding.

\section{Appendices}

\section{A. All Order Tubular Expansion of Vielbein}

In order to derive closed form expressions for the tubular expansion coefficients for vielbein, one starts with the following set of conditions which are equivalent to (2):

$$
e^{(a)}{ }_{B} y^{B}=\bar{e}^{(a)}{ }_{B} y^{B}, \quad y^{A} \omega_{A}{ }^{(b)}{ }_{(c)}=0,
$$

where $e^{(a)}{ }_{b}$ is the vielbein of $L$. Then by making use of the Cartan's structure equations (as was done in [20]) one derives the following second-order differential equation [19]:

$$
\mathbf{d}(\mathbf{d}+\stackrel{b}{\epsilon}) e^{(a)}{ }_{b}(x, y)=\rho^{(a)}{ }_{(c)}(x, y ; y) e^{(c)}{ }_{b}(x, y),
$$

where for any function $f(x, y)$ we have defined

$$
\mathbf{d} f(x, y)=y^{A} \partial_{A} f(x, y) .
$$

Furthermore,

$$
\begin{gathered}
\rho_{(b)}^{(a)}(x, y ; \tilde{y}) \equiv r_{C D(b)}^{(a)}(x, y) \widetilde{y}^{C} \tilde{y}^{D}, \\
\stackrel{b}{\epsilon}= \begin{cases}1 & \text { when } b=B, \\
-1 & \text { otherwise. }\end{cases}
\end{gathered}
$$

The solution to (A.2) can be given in the form of the following tubular expansion [19]:

$$
\begin{aligned}
e^{(a)}= & \sum_{n=0}^{\infty} \sum_{s_{1}, \ldots, s_{n}=0}^{\infty} \mathscr{F}_{\perp}^{(n)}\left(s_{1}, s_{2}, \ldots, s_{n}\right) \\
& \times\left[(y \cdot \mathscr{D})^{s_{1}} \rho(x, 0 ; y) \cdots(y \cdot \mathscr{D})^{s_{n}} \rho(x, 0 ; y)\right]_{\left({ }_{(b)} e_{0}^{(a)},\right.}^{(b)}, \\
e^{(a)}= & \sum_{n=0}^{\infty} \sum_{s_{1}, \ldots, s_{n}=0}^{\infty} \mathscr{F}_{\|}^{(n)}\left(s_{1}, s_{2}, \ldots, s_{n}\right) \\
& \times\left[(y \cdot \mathscr{D})^{s_{1}} \rho(x, 0 ; y) \cdots(y \cdot \mathscr{D})^{s_{n}} \rho(x, 0 ; y)\right]_{(b)}^{(a)} e_{0}^{(b)},
\end{aligned}
$$

where

$$
\begin{gathered}
\mathscr{F}_{\perp}^{(n)}\left(s_{1}, s_{2}, \ldots, s_{n}\right) \\
=\frac{C_{s_{1}}^{s_{1}+s_{2}+\cdots+s_{n}+2 n-1} C_{s_{2}}^{s_{2}+s_{3}+\cdots+s_{n}+2 n-3} \cdots C_{s_{n}}^{s_{n}+1}}{\left(s_{1}+s_{2}+\cdots+s_{n}+2 n+1\right) !}, \\
\mathscr{F}_{\|}^{(n)}\left(s_{1}, s_{2}, \ldots, s_{n}\right)=\frac{C_{s_{1}}^{s_{1}+s_{2}+\cdots+s_{n}+2 n-2} C_{s_{2}}^{s_{2}+s_{3}+\cdots+s_{n}+2 n-4} \cdots 1}{\left(s_{1}+s_{2}+\cdots+s_{n}+2 n\right) !} .
\end{gathered}
$$

$C_{r}^{n}$ are the binomial coefficients and

$$
\begin{aligned}
& {\left[(y \cdot \mathscr{D})^{s} \rho(x, 0 ; y)\right]^{(a)}{ }_{(b)}} \\
& \quad=\mathscr{D}_{A_{1}} \cdots \mathscr{D}_{A_{s}} r^{(a)}{ }_{C D(b)}(x, 0) y^{A_{1}} \cdots y^{A_{s}} y^{C} y^{D},
\end{aligned}
$$


where $\mathscr{D}_{a}$ is the covariant derivative in $L$ with respect to the metric $g_{a b}$. Notice that all such derivatives are evaluated at the submanifold. Finally (recall, according to our rule for notation, $\left.\bar{\omega}_{\alpha}{ }^{(b)}{ }_{C}=\omega_{\alpha}{ }^{(b)}{ }_{C}(x, y=0)\right)$,

$$
\begin{gathered}
e_{0}^{(a)}{ }_{B}=\delta^{(a)}{ }_{B}, \\
e_{0}^{(a)}= \begin{cases}E_{\beta}^{(\alpha)}{ }_{\beta}+\bar{\omega}_{\beta}^{(\alpha)}{ }_{C} y^{C}, & \text { for } a=\alpha, \\
\bar{\omega}_{\beta}{ }^{(A)}{ }_{C} y^{C}, & \text { otherwise, }\end{cases}
\end{gathered}
$$

$E^{(\alpha)}{ }_{\beta}(x)$ being the vielbein of the induced metric on $M$,

$$
\bar{g}_{\alpha \beta}=G_{\alpha \beta}(x)=E_{\alpha}^{(\gamma)}(x) E_{(\gamma) \beta}(x) .
$$

Using the results in (A.5) we find the metric expansion given in (3).

\section{B. Tubular Expansion of Hamiltonian}

Here we will present the detailed computations required to carry out various steps of performing semiclassical expansion as defined in Section 3.1. The rescaling of wavefunction that takes us to the submanifold based description is given by

$$
\psi(z)=\left(\frac{g}{G}\right)^{1 / 4} \psi^{\prime}(z) .
$$

This leads to the following expression for $\mathscr{H}^{\text {sub }}$ as defined in (6)

$$
\mathscr{H}^{\mathrm{sub}}=-\frac{\hbar^{2}}{2}\left(\mathscr{D}^{2}+\mathscr{T}+\mathscr{U}\right)+V
$$

where

$$
\begin{gathered}
\mathscr{T}=-\frac{1}{2} g^{a b} l_{a} \partial_{b}, \quad \mathcal{U}=\frac{1}{16} g^{a b} l_{a} l_{b}+\frac{1}{4}\left(-g^{a b} q_{a b}+\gamma^{c} l_{c}\right), \\
l_{a}=\partial_{a} \ln \left(\frac{g}{G}\right), \quad q_{a b}=\partial_{a} \partial_{b} \ln \left(\frac{g}{G}\right) .
\end{gathered}
$$

The contribution at $O\left(y^{n}\right)$ in the tubular expansion of $\mathscr{H}^{\text {sub }}$ is given by

$$
\mathscr{H}_{n}^{\text {sub }}=-\hbar^{2}\left(K_{n}^{\|}+K_{n}+K_{n}^{\perp}\right)+V_{n}
$$

where

$$
\begin{gathered}
K_{n}^{\|}=\frac{1}{2}\left(\mathbb{D}_{n}^{\|}-d_{n}^{\|}+t_{n}^{\|}+\mathscr{U}_{n}\right), \\
K_{n}=\frac{1}{2}\left(2 \mathbb{D}_{n}-d_{n}+t_{n}\right), \\
K_{n}^{\perp}=\frac{1}{2} \mathbb{D}_{n}^{\perp},
\end{gathered}
$$

and (given a geometric quantity $X$, the notation $X_{n}$ has been explained in (7)),

$$
\begin{gathered}
\mathbb{D}_{n}^{\|}=g_{n}^{\alpha \beta} \partial_{\alpha} \partial_{\beta}, \quad \mathbb{D}_{n}=g_{n}^{\alpha B} \partial_{\alpha} \partial_{B}, \quad \mathbb{D}_{n}^{\perp}=g_{n}^{A B} \partial_{A} \partial_{B}, \\
d_{n}^{\|}=\gamma_{n}^{\alpha} \partial_{\alpha}, \quad d_{n}=\gamma_{n}^{A} \partial_{A}, \\
t_{n}^{\|}=-\frac{1}{2} \sum_{m=0}^{n}\left(g_{n-m}^{\alpha \beta} l_{m \alpha}+g_{n-m}^{A \beta} l_{m A}\right) \partial_{\beta}, \\
t_{n}=-\frac{1}{2} \sum_{m=0}^{n}\left(g_{n-m}^{\alpha B} l_{m \alpha}+g_{n-m}^{A B} l_{m A}\right) \partial_{B} .
\end{gathered}
$$

Finally, the contribution at $O\left(\hbar^{n / 2}\right)$ in the semiclassical expansion of $\mathscr{H}$ works out to be (given $X$, the notation ${ }^{\epsilon} X$ has been defined in (13))

$$
{ }^{\epsilon} \mathscr{H}^{(n)}=-{ }^{\epsilon} K_{n-2}^{\|}-{ }^{\epsilon} K_{n-1}-{ }^{\epsilon} K_{n}^{\perp}+{ }^{\epsilon} V_{n+2} .
$$

This shows how the contribution at a given order in $\hbar$ is related to terms in tubular expansion of various geometric quantities at different orders.

\section{A Note on Loop Space and LSQM}

The loop space $\mathscr{L} \mathscr{M}$ associated with a Riemannian manifold $\mathscr{M}$ is the space of all smooth maps from a parametrized loop to $\mathscr{M}$

$$
\mathscr{L} \mathscr{M}=C^{\infty}\left(s^{1}, \mathscr{M}\right)
$$

Here we will briefly note down some general features of $\mathscr{L} \mathscr{M}$ and LSQM that are relevant for our discussion in this paper. The above definition implies that given any element $l \in \mathscr{L} \mathscr{M}$, there exists a smooth function $p^{(l)}: S^{1} \rightarrow \mathscr{M}$. We wish to define a general coordinate system in $\mathscr{L} \mathscr{M}$ in an open neighborhood of small loops as defined in Section 4.1. To this end we recall the definition of all the open sets as given below (25). Therefore, given any $l \in C\left(U_{\mathscr{M}}\right)$, the entire image $p^{(l)}$ resides inside $\widetilde{U}_{\mathscr{M}}$. Let $x: \widetilde{U}_{\mathscr{M}} \rightarrow \mathbb{R}^{D}$ be a local coordinate system in $\widetilde{U}_{\mathscr{M}} \subset \mathscr{M}$. Then $Z^{(l)}=x \circ p^{(l)}: S^{1} \rightarrow \mathbb{R}^{D}$, and therefore is an element of $\mathscr{L} \mathbb{R}^{D}$ which is the model space of $\mathscr{L} \mathscr{M}$. We consider $Z: C\left(U_{\mathscr{M}}\right) \rightarrow \mathscr{L} \mathbb{R}^{D}$, such that $Z \circ l=Z^{(l)} \in \mathscr{L} \mathbb{R}^{D}$, to be the coordinate functions in the relevant neighborhood in $\mathscr{L} \mathscr{M}$.

Following [14, 15], we will work with a Fourier space representation of these coordinate functions. In this representation the general coordinates of a point $l$ as considered above in $\mathscr{L} \mathscr{M}$ are given by

$$
z^{a}=\oint Z^{\alpha}(\sigma) e^{-i \mathrm{a} \sigma}, \quad \mathrm{a} \in \mathbb{Z}
$$

where $\sigma$ parametrizes the loop, $\oint \equiv \int(d \sigma / 2 \pi)$, and the loop embedding $Z^{\alpha}(\sigma)$ ( $\alpha$ being a target space index) is obtained by following the above definition. We adopt the following convention for an infinite-dimensional coordinate index. It 
is given by a lower case Latin alphabet, which in turn is associated with a pair containing the corresponding Greek alphabet (i.e., a target space index) and an integer, denoted by the same small Latin alphabet in text format. For example, $a \rightarrow(\alpha, \mathrm{a}), b \rightarrow(\beta, \mathrm{b})$. We will also adopt a similar association between such a pair and the corresponding upper case Latin alphabet when the integer is nonzero, that is, $A \rightarrow$ $(\alpha, \mathrm{a}), B \rightarrow(\beta, \mathrm{b})$, and so forth, only when $\mathrm{a}, \mathrm{b} \neq 0$. We use this type of notation in all our discussion involving an explicit coordinate system in $\mathscr{L} \mathscr{M}$.

Reparametrization of the loop corresponds to an isometry of the loop space which exists irrespective of the isometries of $\mathscr{M}$. The generator of this isometry is given by

$$
v^{a}(z)=\oint \partial_{\sigma} Z^{\alpha}(\sigma) e^{-i \mathrm{a} \sigma}
$$

which satisfies the Killing vector equation in $\mathscr{L} \mathscr{M}[10,11]$

$$
\mathscr{D}_{a} v_{b}+\mathscr{D}_{b} v_{a}=0
$$

where $\mathscr{D}_{a}$ is the covariant derivative on $\mathscr{L} \mathscr{M}$. The metric and affine connection on $\mathscr{L} \mathscr{M}$ are given by

$$
\begin{gathered}
g_{a b}(z)=\oint G_{\alpha \beta}(Z(\sigma)) e^{i(\mathrm{a}+\mathrm{b}) \sigma}, \\
\gamma_{b d}^{a}(z)=\oint \Gamma_{\beta \delta}^{\alpha}(Z(\sigma)) e^{i(-\mathrm{a}+\mathrm{b}+\mathrm{d}) \sigma},
\end{gathered}
$$

respectively, where $G_{\alpha \beta}$ and $\Gamma_{\beta \delta}^{\alpha}$ are the metric and affine connection on $\mathscr{M}$, respectively. Notice from (C.3) that the submanifold of vanishing loops, which is given by

$$
z^{A}=\oint Z^{\alpha}(\sigma) e^{-i \mathrm{a} \sigma}=0, \quad \forall \mathrm{a} \neq 0,
$$

is where the Killing vector field vanishes. This situation is similar to the consideration of Kobayashi's theorem in [37] (in finite dimensions), which claims that the space of fixed points of an isometry is a totally geodesic submanifold of even codimension. We will see in Section 4 that the submanifold of interest is indeed totally geodesic. Although, this has infinite number of transverse directions, from the discussion of the infinite dimensional coordinate index done below (C.2), it is clear that for every transverse index $A \rightarrow(\alpha, \mathrm{a})$, there is a pair $\bar{A} \rightarrow(\alpha,-a)$.

We now briefly recall the structure of LSQM following $[14,15]$. The classical NLSM Lagrangian on a flat worldsheet takes the following form in terms of the general coordinates in $\mathscr{L} \mathscr{M}$ :

$$
\begin{gathered}
L=K-V^{\mathscr{L} \mathscr{M}}, \\
K=\frac{1}{2} g_{a b}(z) \dot{z}^{a} \dot{z}^{b}, \\
V^{\mathscr{L} \mathscr{M}}=\frac{1}{2} g_{a b}(z) v^{a}(z) v^{b}(z),
\end{gathered}
$$

where a dot indicates derivative with respect to the worldsheet time. Notice that the potential is proportional to the norm-square of the Killing vector field discussed above. LSQM $[14,15]$ is a formal $\hbar$-deformation of this classical system obtained by following DeWitt's argument in [38]. Therefore, it has the same mathematical structure as that of the toy model discussed in Section 3 with the configuration space replaced by the infinite dimensional loop space (Though a crucial difference is that LSQM should be viewed as a worldline-type description of a relativistic system. See Section 5 for more details on this.). In particular, the matrix element of the Hamiltonian between two scalar states is given by the same equation as in (5), with various quantities now interpreted in the context of $\mathscr{L} \mathscr{M}$ instead of $L$. For example, $\mathscr{D}^{2}$ denotes the Laplacian in $\mathscr{L} \mathscr{M}$.

\section{Existence of $\left(x^{\prime}, y^{\prime}\right)$-System}

In the discussion of Section 4.2.1 we assumed that starting from the direct product coordinate system $\bar{z}=\left(x_{1}, x_{2}\right)$ (see (28), (29)) on $\mathscr{M} \times \mathscr{M}$ one can arrive at another, namely, $z^{\prime}=\left(x^{\prime}, y^{\prime}\right)$, such that the transformed vielbein components are given, up to a constant conformal transformation, by (32) up to quadratic order in $y^{\prime}$. Here we will explicitly construct $z^{\prime}$ in a region whose overlap with the diagonal submanifold is sufficiently small.

We begin by discussing geodesics of $\mathscr{M} \times \mathscr{M}$. These are direct products of geodesics in the two copies of $\mathscr{M}$. The ones which pass through $\bar{z}_{0}=\left(x_{0}, x_{0}\right) \in \Delta_{U_{\mathscr{M}} \times, \mathbb{U}}\left(=U_{\mathscr{M} \times \mathscr{M}} \cap \Delta\right) \mathrm{can}$ be labelled by a unit vector $((1 / \sqrt{2}) \eta,(1 / \sqrt{2}) \zeta) \in T_{x_{0}}(\mathscr{M}) \times$ $T_{x_{0}}(\mathscr{M})$, where $\eta^{\alpha_{1}}\left(\zeta^{\alpha_{2}}\right)$ is the unit tangent to the geodesic in the first copy (second copy) at $x_{1}=x_{0}\left(x_{2}=x_{0}\right)$. The vectors $((1 / \sqrt{2}) \eta,(1 / \sqrt{2}) \eta)$, and $((1 / \sqrt{2}) \eta,-(1 / \sqrt{2}) \eta)$ are parallel and transverse to $\Delta_{U_{\text {IIX II }}}$ at $\left(x_{0}, x_{0}\right)$, respectively. A geodesic whose unit tangent vector at $\left(x_{0}, x_{0}\right)$ is of the form $((1 / \sqrt{2}) \eta,(1 / \sqrt{2}) \eta)$ remains on the diagonal submanifold for ever. This implies that $\Delta$ is a totally geodesic submanifold of $\mathscr{M} \times \mathscr{M}$. We would like to construct $z^{\prime}$ such that $y^{\prime}$ is a geodesic coordinate along the transverse direction given by a unit vector of the form $((1 / \sqrt{2}) \eta,-(1 / \sqrt{2}) \eta)$.

We first consider the following coordinate transformation:

$$
\bar{z}^{a} \longrightarrow \widetilde{z}^{a}=\left(y_{1}^{\alpha_{1}}, y_{2}^{\alpha_{2}}\right),
$$

such that

$$
x_{1}^{\alpha_{1}}=\exp _{x_{0}}^{\alpha_{1}}\left(y_{1}\right), \quad x_{2}^{\alpha_{2}}=\exp _{x_{0}}^{\alpha_{2}}\left(y_{2}\right)
$$

where the exponential map $\exp _{x_{0}}: T_{x_{0}} \mathscr{M} \rightarrow \mathscr{M}$ is given by

$$
\exp _{x_{0}}^{\alpha}(\xi)=x_{0}^{\alpha}+\xi^{\alpha}-\frac{1}{2} \Gamma_{\beta_{1} \beta_{2}}^{\alpha}\left(x_{0}\right) \xi^{\beta_{1}} \xi^{\beta_{2}}+\cdots
$$

$\Gamma_{\beta_{1} \beta_{2}}^{\alpha}$ being the Christoffel symbols of $\mathscr{M}$. We readily recognize that $y_{1}$ and $y_{2}$ are individually $\mathrm{RNC}_{x_{0}}$ in the two copies of $\mathscr{M}$ and the system $\widetilde{z}$ is an $\operatorname{RNC}_{\left(x_{0}, x_{0}\right)}$ in $\mathscr{M} \times \mathscr{M}$. 
The transformed vielbein components are expanded up to quadratic order as [20]

$$
\begin{gathered}
\tilde{e}_{\beta_{1}}^{\left[\alpha_{1}\right]}(\widetilde{z})=E_{\beta_{1}}^{\left(\alpha_{1}\right)}\left(x_{0}\right)+\frac{1}{6} \check{R}^{\left(\alpha_{1}\right)}{ }_{\gamma_{1} \delta_{1} \beta_{1}}\left(x_{0}\right) y_{1}^{\gamma_{1}} y_{1}^{\delta_{1}}, \\
\tilde{e}_{\beta_{2}}^{\left[\alpha_{1}\right]}(\widetilde{z})=0, \\
\tilde{e}^{\left[\alpha_{2}\right]}(\widetilde{z})=0, \\
\tilde{e}_{\beta_{1}}^{\left[\alpha_{2}\right]}(\widetilde{z})=E_{\beta_{2}}^{\left(\alpha_{2}\right)}\left(x_{0}\right)+\frac{1}{6} \check{R}_{\gamma_{2} \delta_{2} \beta_{2}}^{\left(\alpha_{2}\right)}\left(x_{0}\right) y_{2}^{\gamma_{2}} y_{2}^{\delta_{2}} .
\end{gathered}
$$

Next we define the orthonormal frames which are parallel and transverse to the diagonal by giving the following rotation in the local Lorentz frame:

$$
\begin{aligned}
& \tilde{e}_{b}^{[\alpha]}=\frac{1}{\sqrt{2}}\left(\tilde{e}^{\left[\tilde{\alpha}_{1}=\alpha\right]}{ }_{b}+\widetilde{e}^{\left[\alpha_{2}=\alpha\right]}{ }_{b}\right), \\
& \tilde{e}_{b}^{[\hat{\alpha}]}=\frac{1}{\sqrt{2}}\left(\widetilde{e}^{\left[\alpha_{1}=\widehat{\alpha}\right]}{ }_{b}-\widetilde{e}^{-\left[\alpha_{2}=\widehat{\alpha}\right]}{ }_{b}\right),
\end{aligned}
$$

where $\alpha, \widehat{\alpha}=1, \ldots, D$. The parallel and transverse coordinates, $u^{\alpha}$ and $y^{\prime \prime \widehat{\alpha}}$, respectively, are defined by yet another coordinate transformation,

$$
u^{\alpha}=\frac{1}{2}\left(y_{1}^{\alpha_{1}=\alpha}+y_{2}^{\alpha_{2}=\alpha}\right), \quad y^{\prime \prime \widehat{\alpha}}=\frac{1}{2}\left(y_{1}^{\alpha_{1}=\widehat{\alpha}}-y_{2}^{\alpha_{2}=\widehat{\alpha}}\right) .
$$

Therefore, $u^{\alpha}$ is an $\mathrm{RNC}_{\left(x_{0}, x_{0}\right)}$ of $\mathscr{M} \times \mathscr{M}$ which remains parallel to the submanifold $\Delta$. We combine $u^{\alpha}$ with $x_{0}$ to construct a general coordinate $x^{\prime \prime \alpha}$ on $\Delta$,

$$
x^{\prime \prime \alpha}=\exp _{x_{0}}^{\alpha}(u)
$$

Therefore, we seem to have arrived at a coordinate system $z^{\prime \prime a}=\left(x^{\prime \prime \alpha}, y^{\prime \prime \widehat{\alpha}}\right)$ where $x^{\prime \prime \alpha}$ is a general coordinate system on $\Delta$ and $y^{\prime \prime \widehat{\alpha}}$ is orthogonal to it. However, it has been constructed using exponential map with a fixed base point. Therefore, it is guaranteed to be the right one, that is, the one relevant to FNC, only near the base point $\left(x_{0}, x_{0}\right)$. Now onwards we restrict to a region around this point whose overlap with $\Delta$ is sufficiently small. More precisely, we consider $u$ to be at higher order in smallness with respect to $y^{\prime \prime}$, implying that we neglect terms of order $u y^{\prime \prime}$ and $u^{2}$ with respect to those of order $y^{\prime \prime 2}$. With this approximation the transformed vielbein components in $z^{\prime \prime}$-system are given by

$$
\begin{aligned}
\frac{1}{\sqrt{2}} e_{\beta}^{\prime \prime[\alpha]}\left(z^{\prime \prime}\right)= & E_{\beta}^{(\alpha)}\left(x^{\prime \prime}\right)+\frac{1}{6} \check{R}_{\widehat{\gamma} \hat{\delta} \beta}^{(\alpha)}\left(x^{\prime \prime}\right) y^{\prime \prime \widehat{\gamma}} y^{\prime \prime \widehat{\delta}}, \\
& \frac{1}{\sqrt{2}} e^{\prime \prime[\alpha]}\left(z^{\prime \prime}\right)=0, \\
& \frac{1}{\sqrt{2}} e_{\beta}^{\prime \prime[\widehat{\alpha}]}\left(z^{\prime \prime}\right)=0, \\
\frac{1}{\sqrt{2}} e_{\widehat{\beta}}^{\prime \prime[\widehat{\alpha}]}\left(z^{\prime \prime}\right)= & E_{\widehat{\beta}}^{(\alpha)}\left(x^{\prime \prime}\right)+\frac{1}{6} \check{R}_{\widehat{\gamma} \hat{\delta} \widehat{\beta}}^{(\alpha)}\left(x^{\prime \prime}\right) y^{\prime \prime \widehat{\gamma}} y^{\prime \prime \widehat{\delta}} .
\end{aligned}
$$

The $1 / \sqrt{2}$ factors arise because of the standard constant rescaling of the measure when we go to a diagonal. Now onwards, we will absorb this by applying a constant conformal transformation of the metric.

There is a further coordinate transformation which keeps the form of the expansions in (D.8) invariant within the same region of validity, yet making it more general. This is given by $z^{\prime \prime} \rightarrow z^{\prime}=\left(x^{\prime \alpha}, y^{\prime \widehat{\alpha}}\right)$ such that

$$
x^{\prime \prime \alpha}=x^{\prime \alpha}+\left(q-\frac{1}{6}\right) \check{R}_{\widehat{\gamma} \hat{\delta} \beta}^{\alpha}\left(x^{\prime}\right) y^{\prime \widehat{\gamma}} y^{\prime \widehat{\delta}} x^{\prime \beta}, \quad y^{\prime \prime \widehat{\alpha}}=y^{\prime \widehat{\alpha}},
$$

where $q$ is a real constant. The transformed vielbein components are given by (32).

\section{Acknowledgments}

The author is thankful to A. P. Balachandran, Partha Sarathi Chakraborty, Sumit R. Das, Ghanashyam Date, Rukmini Dey, Debashis Ghoshal, Rajesh Gopakumar, Dileep P. Jatkar, Koushik Ray, B. Sathiapalan, Ashoke Sen, Alfred D. Shapere, and Andrew Stacey for useful discussion, questions, and comments. He is also grateful to Harish-Chandra Research Institute (Allahabad) for hospitality and the organizers of the meetings Quantum Theory and Symmetries 7 (Prague, August 2011) and Indian National Strings Meeting 2011 (New Delhi, December 2011) where preliminary versions of this work were presented. Study of Section 3 and related matters have been facilitated by the use of the mathematical program Cadabra [39-41]. The author is thankful to Kasper Peeters for helpful communication.

\section{References}

[1] C. G. Callan and L. Thorlacius, "Sigma models and string theory," in Particles, Strings and Supernovae, A. Jevicki and C.-I. Tan, Eds., World Scientific, Hackensack, NJ, USA, 1989.

[2] A. A. Tseytlin, "Sigma model approach to string theory," International Journal of Modern Physics A, vol. 4, no. 6, pp. 1257$1318,1989$.

[3] J. Honerkamp, "Chiral multi-loops," Nuclear Physics B, vol. 36, no. 1, pp. 130-140, 1972.

[4] D. Friedan, "Nonlinear models in two $\varepsilon$ dimensions," Physical Review Letters, vol. 45, p. 1057, 1980.

[5] D. H. Friedan, "Nonlinear models in two $+\varepsilon$ dimensions," Annals of Physics, vol. 163, no. 2, pp. 318-419, 1985.

[6] L. Alvarez-Gaumé, D. Z. Freedman, and S. Mukhi, "The background field method and the ultraviolet structure of the supersymmetric nonlinear $\sigma$-model," Annals of Physics, vol. 134, no. 1, pp. 85-109, 1981.

[7] L. Eisenhart, Riemannian Geometry, Princeton University Press, Princeton, NJ, USA, 1965.

[8] E. Witten, "Supersymmetry and Morse theory," Journal of Differential Geometry, vol. 17, no. 4, pp. 661-692, 1982.

[9] E. Witten, "The Index of the Dirac Operator in Loop Space," PUPT-1050.

[10] U. Schreiber, "On deformations of 2d SCFTs," Journal of High Energy Physics, vol. 2004, no. 6, article 058, 2004. 
[11] U. Schreiber, "From loop space mechanics to nonabelian strings," http://arxiv.org/abs/hep-th/0509163.

[12] E. Frenkel, A. Losev, and N. Nekrasov, "Notes on instantons in topological field theory and beyond," Nuclear Physics B, vol. 171, pp. 215-230, 2007.

[13] E. Frenkel, A. Losev, and N. Nekrasov, "Instantons beyond topological theory II," http://arxiv.org/abs/0803.3302.

[14] P. Mukhopadhyay, "On a coordinate independent description of string worldsheet theory," http://arxiv.org/abs/0912.3987.

[15] P. Mukhopadhyay, "DeWitt-Virasoro construction in tensor representations," Advances in High Energy Physics, vol. 2012, Article ID 415634, 33 pages, 2012.

[16] P. G. C. Freund and R. I. Nepomechie, "Unified geometry of antisymmetric tensor gauge fields and gravity," Nuclear Physics B, vol. 199, no. 3, pp. 482-494, 1982.

[17] P. S. Florides and J. L. Synge, "Coordinate conditions in a Riemannian space for coordinates based on a subspace," Proceedings of the Royal Society of London Series A, vol. 323, pp. $1-10,1971$.

[18] A. Gray, Tubes, Progress in Mathematics, Addison-Wesley, New York, NY, USA, 2nd edition, 1990.

[19] P. Mukhopadhyay, "All order covariant tubular expansion," http://arxiv.org/abs/1203.1151.

[20] U. Müller, C. Schubert, and A. E. M. van de Ven, "A closed formula for the Riemann normal coordinate expansion," General Relativity and Gravitation, vol. 31, no. 11, pp. 1759-1768, 1999.

[21] R. A. Marcus, "On the analytical mechanics of chemical reactions. Quantum mechanics of linear collisions," Journal of Chemical Physics, vol. 45, pp. 4493-4499, 1966.

[22] H. Jensen and H. Koppe, "Quantum mechanics with constraints," Annals of Physics, vol. 63, pp. 586-591, 1971.

[23] R. C. T. da Costa, "Constraints in quantum mechanics," Physical Review A, vol. 25, pp. 2893-2900, 1982.

[24] P. Maraner, "A complete perturbative expansion for quantum mechanics with constraints," Journal of Physics A, vol. 28, no. 10, pp. 2939-2951, 1995.

[25] K. A. Mitchell, "Gauge fields and extrapotentials in constrained quantum systems," Physical Review A, vol. 63, no. 4, Article ID 042112, 20 pages, 2001.

[26] G. F. Dell'Antonio and L. Tenuta, "Semiclassical analysis of constrained quantum systems," Journal of Physics A, vol. 37, no. 21, pp. 5605-5624, 2004.

[27] J. Wachsmuth and S. Teufel, "Effective hamiltonians for constrained quantum systems," http://arxiv.org/abs/0907.0351.

[28] J. Wachsmuth and S. Teufel, "Constrained quantum systems as an adiabatic problem," Physical Review A, vol. 82, no. 2, Article ID 022112, 2010.

[29] A. Stacey, “The differential topology of loop spaces," http:// arxiv.org/abs/math/0510097, http://ncatlab.org/nlab/show/ equivariant+tubular+neighbourhoods.

[30] M. Nakahara, Geometry, Topology and Physics, Graduate Student Series in Physics, Taylor \& Francis, Boca Raton, Fla, USA, 2nd edition, 2003.

[31] S. Kobayashi and K. Nomizu, Foundations of Differential Geometry II, Wiley, New York, NY, USA, 1st edition, 1969.

[32] M. V. Berry, "Quantal phase factors accompanying adiabatic changes," Proceedings of the Royal Society of London A, vol. 392, no. 1802 , pp. 45-57, 1984.

[33] S. Sasaki, "On the differential geometry of tangent bundles of Riemannian manifolds," The Tohoku Mathematical Journal, vol. 10, pp. 338-354, 1958.
[34] R. M. Wald, General Relativity, University of Chicago Press, Chicago, Ill, USA, 1984.

[35] A. Kriegl and P. W. Michor, The Convenient Setting of Global Analysis, vol. 53 of Mathematical Surveys and Monographs, American Mathematical Society, Providence, RI, USA, 1997.

[36] J. Polchinski, String Theory. Volume 1: An Introduction to the Bosonic String, Cambridge University Press, Cambridge, UK, 1998.

[37] S. Kobayashi, "Fixed points of isometries," Nagoya Mathematical Journal, vol. 13, pp. 63-68, 1958.

[38] B. S. DeWitt, "Point transformations in quantum mechanics," Physical Review, vol. 85, no. 4, pp. 653-661, 1952.

[39] K. Peeters, "Symbolic field theory with Cadabra," Computeralgebra Rundbrief, vol. 41, p. 16, 2007.

[40] K. Peeters, "Introducing Cadabra: a symbolic computer algebra system for field theory problems," http://arxiv.org/abs/hepth/0701238.

[41] L. Brewin, "A brief introduction to Cadabra: a tool for tensor computations in general relativity," Computer Physics Communications, vol. 181, no. 3, pp. 489-498, 2010. 

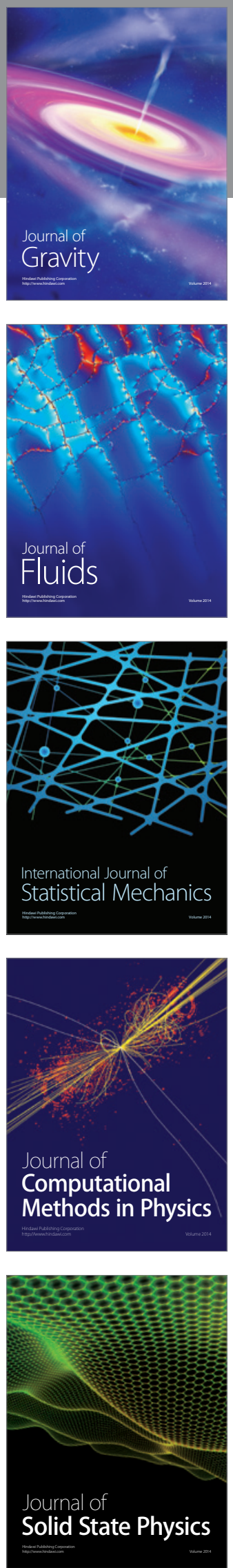

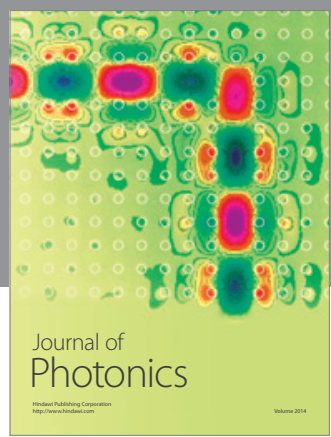

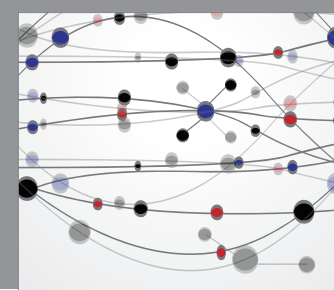

The Scientific World Journal

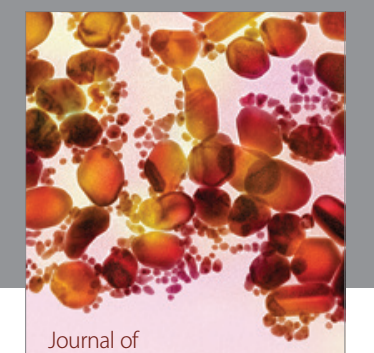

Soft Matter
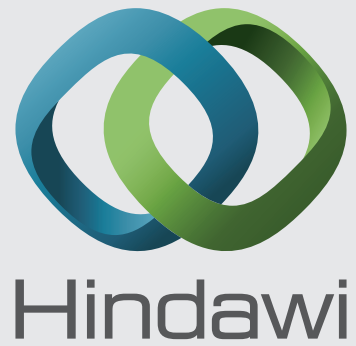

Submit your manuscripts at

http://www.hindawi.com
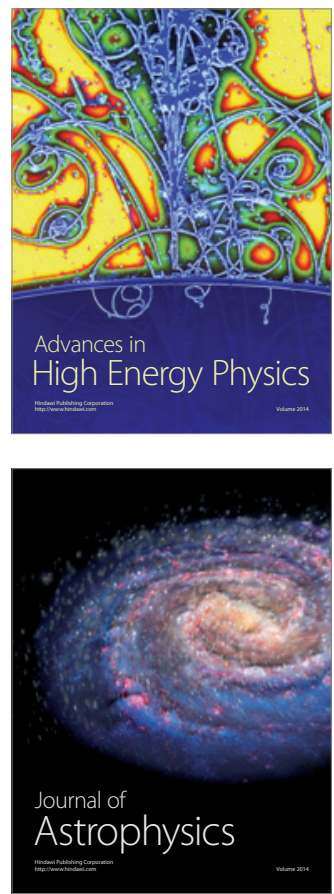
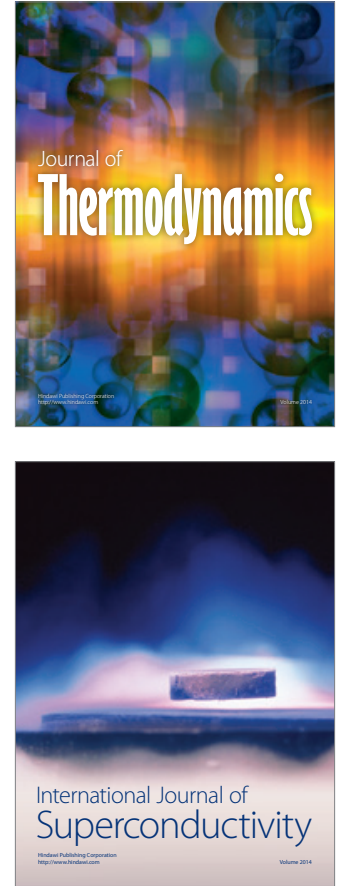
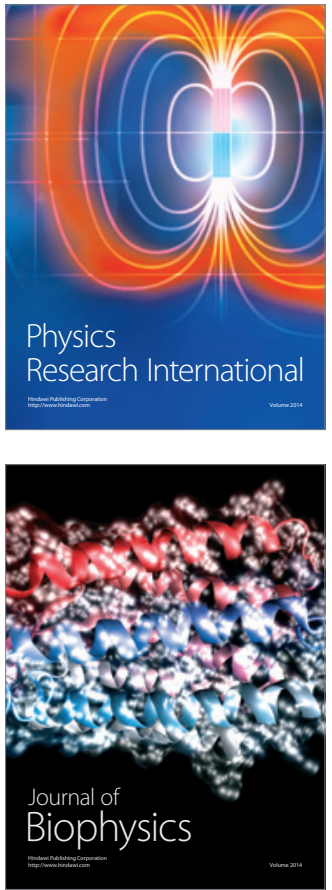
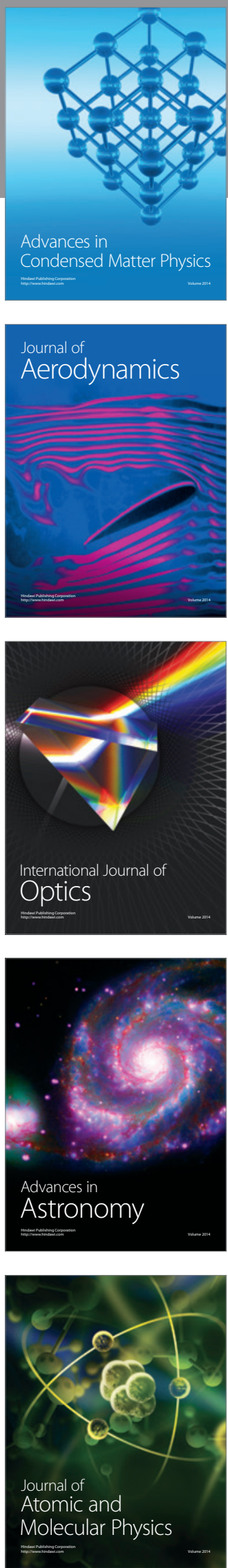